DOI: 10.1002/adom.201801791

Article type: Communication

\title{
Low threshold polariton lasing from a solution processed organic semiconductor in a planar microcavity
}

Sai Kiran Rajendran, Mengjie Wei, Hamid Ohadi, Arvydas Ruseckas, Graham A. Turnbull*, Ifor D. W. Samuel*

Dr. Sai Kiran Rajendran, Mengjie Wei, Dr. Hamid Ohadi, Dr. Arvydas Ruseckas, Prof. Graham A. Turnbull*, Prof. Ifor D. W. Samuel*

Organic Semiconductor Centre, SUPA, School of Physics and Astronomy, University of St Andrews, St Andrews, KY16 9SS United Kingdom

E-mail: gat@st-andrews.ac.uk, idws@,st-andrews.ac.uk

Keywords: strong coupling, organic semiconductor, microcavity, low threshold, polariton lasing

\section{Abstract:}

Organic semiconductor materials have been widely studied for light emission and lasing due to their ability to tune the emission wavelength through chemical structural modification and their relative ease of fabrication. Strong light-matter coupling is a promising route towards a coherent light source because it has the potential for thresholdless polariton lasing. However, the materials studied so far have relatively high thresholds for polariton lasing. Here we report the suitability of pentafluorene for strong coupling and low threshold polariton lasing. We use a protective buffer layer to reduce degradation during fabrication and lower the lasing threshold using negative detuning to maximize radiative decay. We obtain a low threshold of $17 \mu \mathrm{J} \mathrm{cm}^{-2}$, corresponding to an absorbed energy density of $11.7 \mu \mathrm{J} \mathrm{cm}^{-2}$. This study shows that pentafluorene is an attractive material for polariton lasing and will assist in the development of low threshold electrically pumped lasing from polariton devices. 


\section{Introduction}

Strongly coupled light-matter systems have attracted considerable interest due to their possibility to realize low threshold polariton lasing as well as to study quantum systems such as polariton condensation and superfluidity. ${ }^{[1]}$ Only a few inorganic semiconductor materials that exhibit high binding energy have demonstrated room temperature low threshold polariton lasing. ${ }^{[2]}$ Organic semiconductor materials can show robust strong coupling at room temperatures, ${ }^{[3]}$ due to their large exciton binding energy (of several hundreds of meV) and high oscillator strength. They have been widely applied in light emitting devices and conventional lasers, ${ }^{[4]}$ but despite these promising features, it is only recently that polariton lasing has been realized in such materials. ${ }^{[5,6]}$ A growing understanding of the suitability of organic semiconductor materials is now opening up more detailed studies of polariton condensates, including lateral confinement, studies of coherence, stability and superfluidity. ${ }^{[7,8]}$

A major attraction of polariton lasers is the potential to achieve much lower thresholds than in conventional photon lasers, since no population inversion is required. Achieving low threshold operation is vital for future organic polariton devices, both to enhance stability by reducing photo-induced degradation, and to reduce the number of charge carriers required to achieve an electrically pumped polariton laser. However, the cavity polariton lasing thresholds from organic semiconductors in planar microcavities reported to date ${ }^{[5-6]}$ are higher than $300 \mu \mathrm{J} \mathrm{cm}^{-2}$ in incident excitation density and also higher than photon lasing thresholds from the same class of materials. There is therefore a need for new materials that can achieve low threshold polariton lasing. In this paper we report polariton lasing using an oligofluorene in a planar dielectric microcavity giving an order of magnitude reduction in 
threshold to $17 \mu \mathrm{J} \mathrm{cm}^{-2}$. This corresponds to an absorbed energy density of $11.7 \mu \mathrm{J} \mathrm{cm}{ }^{-2}$. It is also comparable with the lowest plasmon exciton polariton lasing threshold of $18 \mu \mathrm{J} \mathrm{cm}^{-2}$, obtained from a dye on a laterally patterned nanoparticle array. ${ }^{[9]}$

To achieve this low-threshold operation, we select a material with high photoluminescence quantum yield (PLQY) and oscillator strength, pentafluorene, and introduce a buffer layer in the top mirror to retain the high PLQY in the dielectric microcavity. We observe the key features of polariton lasing including a non-linear emission intensity beyond a threshold excitation density, a drop in the spectral linewidth of emission, and increase in directionality of emission. An associated collapse in the spatial distribution of emission and macroscopic coherence are also observed. In contrast with photon lasing, accumulation of population is at the bottom of the lower polariton branch. We also observe a drop in polariton lasing threshold by simultaneously increasing the radiative component of the lower polariton mode through negative detuning and the proximity to the spectral gain window of pentafluorene. This suggests that the population mechanism dominating the lower polariton branch is largely radiative. ${ }^{[10]}$

\section{Results and Discussion}

Oligofluorenes are a class of materials associated with high exciton binding energy and absorption oscillator strength. ${ }^{[11,12]}$ They are also associated with very short radiative lifetimes $^{[12,13]}$ and high photoluminescence (PL) quantum yield. ${ }^{[14]}$ Oligofluorene films have been reported not to show green excimer emission when annealed in air as can happen in polymers. ${ }^{[15]}$ Oligofluorene films have also shown amplified spontaneous emission ${ }^{[16]}$ with thresholds of the order of $1 \mu \mathrm{J} \mathrm{cm}^{-2}$. Oligofluorenes can be easily synthesized and processed into films from solution or evaporated to give films of a desired thickness. These properties 
make oligofluorenes suitable materials for organic polariton lasers. In a previous study, we observed that the PL quantum yield increased and the radiative lifetime decreased when the number of fluorene units in oligofluorene increases. ${ }^{[14]}$ The transition dipole moment also increased with the oligomer length. For these reasons, we chose to study pentafluorene in this work.

Pentafluorene has an inhomogenously broadened absorption peak at $369 \mathrm{~nm}(3.36 \mathrm{eV})$ with a FWHM of $60 \mathrm{~nm}(517 \mathrm{meV})$. The emission from neat films present two vibronic peaks at 417 $\mathrm{nm}(2.96 \mathrm{eV})$ and $442 \mathrm{~nm}(2.81 \mathrm{eV})$ with an overall FWHM of $44.5 \mathrm{~nm}(314 \mathrm{meV})$. The energy difference between the peak absorption and peak emission is $400 \mathrm{meV}$. The neat films also present a very short emission lifetime of 440 ps and a high PLQY close to $90 \%$ (see supplementary information for more details). We fabricate strongly coupled pentafluorene in planar microcavities by spin-coating neat films of different thicknesses sandwiched between distributed Bragg reflectors (DBRs) (Figure 1(a)). The DBRs consisted of pairs of $\mathrm{SiO}_{2} / \mathrm{Ta}_{2} \mathrm{O}_{5}$, and were designed with a center reflectivity of $410 \mathrm{~nm}(3.02 \mathrm{eV})$ at normal incidence to include both the peak absorption and the two main emission peaks within the stop band (Figure 1(b)). The bottom DBR bandwidth was also optimized to have peak transmission at $343 \mathrm{~nm}(3.62 \mathrm{eV})$ to allow optimal excitation of the pentafluorene by the 343 $\mathrm{nm}$ laser pulses incident on the bottom DBR. The deposition of the top DBR by radiofrequency $(\mathrm{RF})$ sputtering on pentafluorene would expose the active material to argon and oxygen plasma. Studies on cellulose and polypropylene have shown that cleavage and oxidation of covalent bonds in the material up to $10 \mathrm{~nm}$ from the surface can take place on exposure to RF argon and oxygen plasma. ${ }^{[17]}$ Such functionalization would degrade the light emitting properties of pentafluorene. Hence there is a need to protect the active material using a transparent material with a thickness of about $10 \mathrm{~nm}$. While sputtering cannot be used to 
deposit the protective layer, spin coating is also unsuitable at it would dissolve the active material. Lithium fluoride (LiF) was chosen as an appropriate material that can be evaporated to about $10 \mathrm{~nm}$ thickness on pentafluorene films. We find that sputter deposition on bare pentafluorene reduces the PLQY to $34 \%$, but a LiF protective layer followed by a sputtered layer gives a PLQY of 56\% (see supplementary information S1). The bottom (top) DBRs showed a transmission minimum of $0.8 \%(1.8 \%)$. Using values of $99.2 \%(98.2 \%)$ for reflectivity we calculate a finesse of 290 for the cavities.

Three cavities A, B and C were fabricated with neat pentafluorene active layer films of different thicknesses resulting in polariton modes at $437 \mathrm{~nm}, 432 \mathrm{~nm}$ and $422 \mathrm{~nm}$ respectively at normal incidence. The thicknesses of the films in the three cavities were estimated to be $115 \mathrm{~nm}, 110 \mathrm{~nm}$ and $94 \mathrm{~nm}$ respectively based on transfer matrix calculations of the cavity resonance. Test films deposited under similar conditions later resulted in films between 105 $\mathrm{nm}$ to $76 \mathrm{~nm}$ for similar range of spin speeds. The optical resonances are negatively detuned from the exciton energy at normal angle of incidence. Experimental measurement of the angle dependent reflectivity from cavity $\mathrm{A}$ is shown using scattered points in Figure 1(c). The experimentally recorded reflectivity spectra at small and large angles are shown in supplementary section 2 . An anti-crossing in the dispersion, a characteristic feature of strong coupling, is clearly observed. The dispersion of the lower and upper polariton modes can be matched to a coupled oscillator model whose solutions follow the relation

$$
E_{U, L}(\theta)=1 / 2\left[\left(E_{p}(\theta)+E_{\theta x}\right) \pm \sqrt{\left(E_{p}(\theta)-E_{\theta x}\right)^{2}+4 \Omega^{2}}\right]
$$

for the energy of the upper $\left(\mathrm{E}_{\mathrm{U}}\right)$ and lower polariton $\left(\mathrm{E}_{\mathrm{L}}\right)$ modes, where $E_{\theta x}=3.4 \mathrm{eV}$ is the peak absorption energy of pentafluorene film, $E_{p}(\theta)$ is the angle $(\theta)$ dependent energy of the cavity mode given by 
$E_{p}(\theta)=E_{p}(0) / \sqrt{1-\frac{\sin ^{2} \theta}{n_{e f f}^{2}}}$

$n_{\text {eff }}=1.8$ is the effective refractive index of the cavity, giving a half-Rabi splitting energy $(\Omega)$ of $\sim 250 \mathrm{meV}$. The coupled oscillator model dispersion is plotted with dotted lines in figure 1(c). The dispersion of the pentafluorene microcavities was also calculated using transfer matrix approach. The refractive index of neat pentafluorene film was obtained using fits to experimentally recorded variable angle spectroscopic ellipsometry (see Supplementary Figure S4). The TM polarized reflectivity of the cavity was then calculated for a $115 \mathrm{~nm}$ thick active layer as shown in Figure 1(c) as pseudo color map. Both methods fit excellently with the experimental data. From the coupled oscillator model, the photon fraction of the polariton mode and detuning (energetic separation between the exciton and photon mode) can be extracted (see Supplementary Figure S5). We have $85 \%$ to $75 \%$ photon fraction at the bottom of the lower polariton branch and a negative detuning of $450 \mathrm{meV}, 400 \mathrm{meV}$ and $330 \mathrm{meV}$ for the three cavities.

To measure the lasing characteristics of the pentafluorene microcavities, the angle-resolved polariton emission spectra were recorded in Fourier space by varying the excitation density. ${ }^{[18]}$ The cavities were excited non-resonantly with $343 \mathrm{~nm} 200$ fs duration pulses through the substrate. Figure 2 (a-c) depicts the vertically polarized emission from the cavity at three different incident (absorbed) excitation densities of $0.5 P_{t h}^{A}, 1.5 P_{t h}^{A}$ and $3 P_{\text {th }}^{A}$ where $P_{\text {th }}^{A}=16.7(11.7) \mu \mathrm{J} \mathrm{cm}^{-2}$ (4.0 $\mathrm{nJ}$ pulse energy, $175 \mu \mathrm{m}$ spot diameter). Below threshold the emission follows the lower polariton dispersion obtained in the reflectivity spectra with emission over a large range of angles (Figure 2(a)). The cavity mode obtained from the coupled oscillator model is plotted as yellow dashed lines. The uncoupled dispersionless pentafluorene film emissions at 417 and $442 \mathrm{~nm}$ (vibronic peaks) are weak showing the 
consistency and quality of the fabrication. The absence of these features below threshold indicates that the molecules are strongly coupled and emission occurs only through the polariton mode. Above threshold a spectrally narrow and highly directional emission from the bottom of the lower polariton branch appears, which increases in intensity rapidly with increasing excitation density (Figure 2(b)). This is a key feature of polariton lasing where polaritons of large in-plane wave vector $\left(\mathrm{k}_{\|}\right)$accumulate macroscopically at the bottom of the lower polariton branch. We note that the emission has a narrow divergence and flat dispersion suggesting that it comes from a spatially localized state at a single energy. At $3 P_{t h}^{A}$, the emission extends to higher energy modes due to repulsive interaction between polaritons (Figure 2(c)) similar to inorganic microcavities. ${ }^{[19]}$

Real space images of the emission show the localization effect as intensity increase. At 0.5 $P_{t h}^{A}$ the emitting spot has the same size as the excitation spot showing a Gaussian distribution of polariton emission (Figure 2(d)). As the threshold is crossed the peak emission emerges mainly from localized spots extending a few micrometers in size, as seen in many other materials, ${ }^{[7]}$ (Figure 2(e)). The positions of the spots are repeatable with measurements of the same excitation densities showing that they may be due to inhomogeneity in the film thickness. On increasing the excitation density further the lasing spots spread to a larger area as a result of polariton repulsive interactions (Figure 2(f)).

Cavities B and C show similar emission spectra (see Supplementary FigureS6). However, the incident (absorbed) lasing threshold increases as the detuning reduces giving $P_{t h}^{B}=20 \mu \mathrm{J} \mathrm{cm}{ }^{-2}$ $\left(14 \mu \mathrm{J} \mathrm{cm}^{-2}\right)$ and $P_{\text {th }}^{C}=46 \mu \mathrm{J} \mathrm{cm}^{-2}\left(32.2 \mu \mathrm{J} \mathrm{cm}^{-2}\right)$. This could be attributed to higher losses due to reabsorption of excitations by the uncoupled exciton reservoir, as increasing the cavity 
frequency leads to greater overlap with the molecular absorption spectrum. Despite this, polariton lasing is observed over a wide range of negative detunings in these cavities. The uncoupled exciton reservoir can in principle decay both radiatively as well as non-radiatively into the lower polariton branch. ${ }^{[10]}$ Strongly coupled J-aggregates have been observed to decay predominantly non-radiatively ${ }^{[20]}$ due to their low PLQY. However drop in polariton lasing threshold with the larger PLQY pentafluorene and larger detuning suggest that radiative decay is the dominant polariton relaxation mechanism here. From real space images in both these cavities (Supplemental Figure S6 (d-f) and (j-1)), it is observed that below threshold the spatial polariton distribution follows the excitation spot. Above threshold excitation density, highly populated regions that are spatially fragmented and localized near the excitation spot center appear. With increasing excitation density, polariton repulsive interaction that result in blue shifted emission also result in spatial spread to a larger area.

Figure 3 (a) shows several emitted spectra, collected in the range of $\pm 5^{\circ}\left(\right.$ or $\left.\left|\mathrm{k}_{\|}\right|<1.3 \mu \mathrm{m}^{-1}\right)$, above and below threshold excitation density $\left(P_{t h}^{A}\right)$ for cavity A. We observe that sharp intense peaks appear above threshold that do not shift in energy. At threshold, a narrow peak on the low energy side of the lower polariton branch emerges and increases in intensity with excitation density. Above threshold multiple narrow peaks each of spectral FWHM around $0.2 \mathrm{~nm}$ emerge. We believe that the disorder in layer thickness across the sample leading to variation in resonant photon energy, is the reason for multiple peaks in the emission spectrum above threshold. Figure 3(b) plots the peak emission intensity as a function of incident excitation density (solid symbols). Below threshold the peak intensity increases sublinearly while above the threshold excitation density, the emitted intensity increases superlinearly. For the range of excitation densities up to $3 P_{t h}^{A}$ the emission is repeatable as seen through three consecutive measurements. A second threshold into photon lasing regime was not observed 
as the cavity undergoes irreversible photo-degradation of emission intensity for excitation densities beyond $3 P_{t h}^{A}$. For organic semiconductors in planar microcavities, only green fluorescent protein has been able to show this transition at an excitation density of about 250 $\mathrm{mJ} \mathrm{cm}{ }^{-2}(125 \mathrm{~nJ}$ on $8 \mu \mathrm{m}$ spot$)$ due to its high damage threshold. ${ }^{[5]}$ The spectral FWHM over this angular range of emission is plotted in the same graph using hollow symbols. The emission linewidth at normal incidence below threshold is $1.4 \mathrm{~nm}(9.8 \mathrm{meV})$. Just above threshold the FWHM drops to $0.2 \mathrm{~nm}(1.4 \mathrm{meV})$. With increasing excitation density, due to simultaneous population of narrow modes, the effective FWHM increases. The blue shift of peak spectral emission is an indication of repulsive interaction between polaritons (Figure 3(c)). The blue shift below threshold is negligible compared to the spectral FWHM and hence can be neglected. At threshold, there is a small red shift of peak emission as a result of multiple narrow peaks appearing within the lower polariton mode possibly due to disorder in cavity thickness across the excitation spot. At higher excitation densities, the relative intensities of the multiple narrow peaks changes showing an effective blue shift of $3 \mathrm{meV}$. While the shift is smaller than the effective FWHM due to the multiple modes, it is of similar magnitude to that observed in other organic semiconductor polariton lasers. ${ }^{[5-8]}$ However, each of the narrow peaks do not shift significantly in energy, indicating weak polaritonpolariton interactions.

Similar spectra emitted from cavity B and C are presented in the supplementary figure S7 Below threshold the emission from these cavities has a slightly larger spectral FWHM possibly due to increased inhomogeneity in the film. We also see that above threshold the line shape appears to consist of multiple sharp lines. Once again, each of these narrow modes have a spectral FWHM of about $0.2 \mathrm{~nm}$. However, simultaneous population of these modes result in larger effective spectral FWHM. Both the cavities exhibit lasing from the bottom of 
the lower polariton branch above threshold followed by an effective blue shift of the peak position by about $3 \mathrm{meV}$. The pump energy dependence of the emission intensity is repeatable about the threshold excitation density for all the three cavities.

It was also observed that the polariton emission below threshold was largely unpolarized. The cavities were pumped non-resonantly with respect to the lower polariton branch or bottom of the exciton reservoir in order to allow polarization memory loss. However polariton emission above threshold excitation density had the same polarization as the pump laser. This is due to the scattering rate into the bottom of the lower polariton branch at high excitation densities being much faster than the depolarization time. Details of polariton emission polarization with different pump polarization is given in the supplementary section 6. Similar polarization pinning has been observed in MeLPPP cavities by Plumhof et al. ${ }^{[5]}$

Long range coherence is another indicator of polariton lasing. In order to demonstrate the appearance of long-range coherence, emission from the sample was split in a Mach-Zehnder interferometer with a retroreflector in one arm and interfered spatially and temporally onto a CCD to acquire an interferogram. ${ }^{[18]}$ Figure $3(\mathrm{~d})$ shows an interferogram when cavity $\mathrm{A}$ is excited above threshold density. Fringes are visible up to $5 \mu \mathrm{m}$ in distance showing that long range spatial coherence is present (see supplementary material section 7 for more details). All three cavities show onset of fringes only above threshold excitation density. These fringes are absent below threshold excitation density showing that coherence is not forced on polariton emission by the excitation pulses themselves. We find that similar to inorganic microcavities multiple spatially separated condensates can appear which then phase-lock as excitation power is increased. ${ }^{[21]}$ The results provide clear evidence of macroscopic coherence from the emitting spots. 
In conclusion, pentafluorene was identified as a promising material for room temperature polaritonics. In particular it was found to give strong coupling and low threshold polariton lasing due to its strong absorption as well as high PLQY. Our results are compared with thresholds of other cavity polariton lasers in Table I. The table shows the thresholds of organic and inorganic semiconductor materials that have been reported to show room temperature polariton lasing in microcavities. Among organic materials, pentafluorene has the lowest incident excitation threshold by an order of magnitude, and is less than half of the lowest reported absorbed energy density. Inorganic semiconductor materials also present a comparable low threshold but generally require more complex fabrication techniques. It may be possible to reduce the polariton lasing threshold further in pentafluorene by either optimizing the protective layer or changing the photonic confinement geometry to a zerodimensional cavity or a surface plasmon coupled structure. In this work, we showed that the active layer can be deposited by spin coating and that by using an appropriate protective layer, a top DBR can be sputter deposited without significant degradation. Strong coupling in pentafluorene cavities was achieved exhibiting a Rabi splitting energy of $500 \mathrm{meV}$. Low threshold polariton lasing was achieved in three differently detuned pentafluorene cavities. It was observed that the threshold reduces in cavities with larger negative detuning and this was assigned to be due to a higher radiative decay component of the polariton mode. On excitation with a large spot, we see fragmentation into several bright spots. They are found to be coherent with each other even through separated by a few microns distance. This study significantly lowers the polariton lasing thresholds demonstrated so far with strongly coupled organic semiconductors in planar microcavities and so brings electrical pumping of such devices closer. 


\section{WILEY-VCH}

\section{Experimental section}

The absorption spectrum of the pentafluorene film was recorded with a UV-visible spectrometer CARY 300 and steady state PL spectrum was recorded using an Edinburgh Instruments FLS 980 fluorimeter with 343 nm excitation from a Xenon lamp. The complex refractive index of pentafluorene film were determined by spin coating the film on a silicon substrate and performing variable angle spectroscopic ellipsometry. Using WVASE32 software the data were first fitted in the transparent region to determine the parameters for a Cauchy model. These parameters were then fixed and a multiple regression fit was performed to obtain the complex refractive index of the film over the entire range of the UV-visible spectrum.

The DBR mirrors were RF sputter deposited in an Angstrom Engineering deposition system. Alternating layers of $46.5 \mathrm{~nm} \mathrm{Ta}_{2} \mathrm{O}_{5}$ and $69.7 \mathrm{~nm} \mathrm{SiO}_{2}, 10.5$ pairs in total, followed by $10 \mathrm{~nm}$ $\mathrm{SiO}_{2}$ were sputtered on quartz substrates, to fabricate the bottom distributed Bragg reflector (DBR). Pentafluorene, purchased from American Dye Source Inc. ADS056FO, was dissolved in toluene (99.8\% anhydrous, Sigma Aldrich) at a concentration of $22 \mathrm{mg} / \mathrm{ml}$ and left stirring overnight at $323 \mathrm{~K}$. It was then sonicated in an ultrasonic bath for 1 minute and spin-coated on the DBRs. A range of spin speeds were used to form films of different thicknesses in a nitrogen glove box environment. The thickness of the active layer in the cavities were estimated using spectroscopic ellipsometric measurements of similarly deposited films on glass substrates. A $10 \mathrm{~nm}$ thick LiF layer was then evaporated on the organic film to protect to protect it from plasma during subsequent top DBR sputtering process. The top DBR consisted of $10 \mathrm{~nm} \mathrm{SiO} 2$ followed by 6.5 pairs of alternating $46.5 \mathrm{~nm} \mathrm{Ta}_{2} \mathrm{O}_{5}$ and $69.7 \mathrm{~nm} \mathrm{SiO}_{2}$. Angle resolved reflectivity spectra for angles from $0^{\circ}$ to $40.5^{\circ}$ were measured using Fourier plane imaging with a 0.65 numerical aperture Nikon microscope objective onto the slit of a spectrometer (Andor 500SR coupled to an IDus 420 CCD or Newton 940 CCD). The angle resolved reflectivity in the range of $42^{\circ}$ to $75^{\circ}$ was recorded using a J. A. Woollam VASE 


\section{WILEY-VCH}

ellipsometer with the white light focused on to a $200 \mu \mathrm{m}$ diameter spot. For polariton lasing studies, $200 \mathrm{fs}$ duration pulses of $343 \mathrm{~nm}$ wavelength generated from the third harmonic of a femtosecond laser (Pharos from Light Conversion), were focused on the microcavities using a $250 \mathrm{~mm}$ focal length lens from the substrate side. The emission was collected using the Nikon objective from the top DBR side and angle resolved again by Fourier plane imaging. The real space images of the emitting spot were also recorded on the same CCD spectrometer with the zeroth order of the grating. The excitation spot size was measured using a beam profilometer (Lasercam HR from Coherent) to be $175 \mu \mathrm{m}$ at the sample plane. The magnification of the real space imaging system was estimated using a Thorlabs high resolution target (R2L2S1N1). For lasing studies, the incident excitation density was varied using a neutral density filter and the averaged incident energy per pulse was calculated using the average excitation power measured using a photodiode sensor head (Thorlabs S170C coupled to PM100D console) and repetition rate. The angle resolved emission was collected by the Andor spectrograph at each excitation density for 0.5 secs with the excitation repetition rate at $5 \mathrm{kHz}$ or $1 \mathrm{kHz}$. For macroscopic coherence measurements, reflection geometry was used for excitation and emission detection with a Nikon 0.65 numerical aperture objective in order to create a small number of closely spaced lasing spots. The collected emission was split into two arms of an interferometer that directed the reflected and retro-reflected image into the Andor CCD spectrograph.

\section{Supporting Information}

A supplementary document with supporting text and figures are available from Wiley Research Online and from the author. Research data supporting this publication is available at https://doi.org/10.17630/5503c28f-dea7-4a6e-afca-a6a2e89275d3 


\section{WILEY-VCH}

\section{Acknowledgements}

We are grateful to EPSRC for support through the hybrid polaritonics program grant

(EP/M025330/1). We are also thankful to Jonathan Keeling, University of St Andrews, for useful discussions towards the interpretation of the results. S. K. R. acknowledges valuable help from Laura Tropf, University of St Andrews, regarding sputter deposition and Simon Betzold, Christof Dietrich and Sven Höfling, Universität Würzburg, for support with the experimental setup.

Received: ((will be filled in by the editorial staff))

Revised: ((will be filled in by the editorial staff)) Published online: ((will be filled in by the editorial staff)) 


\section{WILEY-VCH}

\section{References}

[1] (a) A. Imamoğlu, R. J. Ram, S. Pau, Y. Yamamoto, Phys. Rev. A - At. Mol. Opt. Phys. 1996, 53, 4250; (b) H. Deng, H. Haug, Y. Yamamoto, Rev. Mod. Phys. 2010, 82, 1489; (c) A. Amo, J. Lefrère, S. Pigeon, C. Adrados, C. Ciuti, I. Carusotto, R. Houdré, E. Giacobino, A. Bramati, Nat. Phys. 2009, 5, 805.

[2] (a) S. Christopoulos, G. B. H. Von Hogersthal, A. J. D. Grundy, P. G. Lagoudakis, A. V. Kavokin, J. J. Baumberg, G. Christmann, R. Butte, E. Feltin, J. F. Carlin, N. Grandjean, Phys. Rev. Lett. 2007, 98, 126405; (b) G. Christmann, R. Butt, E. Feltin, J. F. Carlin, N. Grandjean, Appl. Phys. Lett. 2008, 93, 1; (c) A. Das, J. Heo, M. Jankowski, W. Guo, L. Zhang, H. Deng, P. Bhattacharya, Phys. Rev. Lett. 2011, 107, 066405; (d) T.-C. Lu, Y.-Y. Lai, Y.-P. Lan, S.-W. Huang, J.-R. Chen, Y.-C. Wu, W.-F. Hsieh, H. Deng, Opt. Express 2012, 20, 5530; (e) R. Su, C. Diederichs, J. Wang, T. C. H. Liew, J. Zhao, S. Liu, W. Xu, Z. Chen, Q. Xiong, Nano Lett. 2017, 17, 3982.

[3] (a) D. G. Lidzey, D. D. C. Bradley, M. S. Skolnick, T. Virgili, S. Walker, D. M. Whittaker, Nature 1998, 395, 53; (b) R. J. Holmes, S. R. Forrest, Org. Electron. 2007, 8, 77. [4] (a) K. Müllen, U. Scherf, Organic Light Emitting Devices: Synthesis, Properties and Applications, John Wiley \& Sons, 2006; (b) I. D. W. Samuel, G. A. Turnbull, Chem. Rev. 2007, 107, 1272.

[5] (a) S. Kéna-Cohen, S. R. Forrest, Nat. Photonics 2010, 4, 371; (b) J. D. Plumhof, T. Stöferle, L. Mai, U. Scherf, R. F. Mahrt, Nat. Mater. 2014, 13, 247; (c) C. P. Dietrich, A. Steude, L. Tropf, M. Schubert, N. M. Kronenberg, K. Ostermann, S. Höfling, M. C. Gather, Sci. Adv. 2016, 2, e1600666; (d) T. Cookson, K. Georgiou, A. Zasedatelev, R. T. Grant, T. Virgili, M. Cavazzini, F. Galeotti, C. Clark, N. G. Berloff, D. G. Lidzey, P. G. Lagoudakis, Adv. Opt. Mater. 2017, 5, 1.

[6] K. S. Daskalakis, S. A. Maier, R. Murray, S. Kéna-Cohen, Nat. Mater. 2014, 13, 271.

[7] (a) D. Sanvitto, S. Kéna-Cohen, Nat. Mater. 2016, 15, 1061; (b) F. Scafirimuto, D.

Urbonas, U. Scherf, R. F. Mahrt, T. Stöferle, ACS Photonics 2018, 5, 85; (c) K. S. Daskalakis, S. A. Maier, S. Kéna-Cohen, Phys. Rev. Lett. 2015, 115, 1; (d) G. Lerario, A. Fieramosca, F. Barachati, D. Ballarini, K. S. Daskalakis, L. Dominici, M. De Giorgi, S. A. Maier, G. Gigli, S. Kéna-Cohen, D. Sanvitto, Nat. Phys. 2017, 13, 837.

[8] N. Bobrovska, M. Matuszewski, K. S. Daskalakis, S. A. Maier, S. Kéna-Cohen, ACS Photonics 2018, 5, 111.

[9] M. Ramezani, A. Halpin, A. I. Fernandez-Dominguez, J. Feist, S. R. K. Rodriguez, F. J. García-Vidal, J. G. Rivas, Optica 2017, 4, 31.

[10] (a) M. Litinskaya, P. Reineker, V. M. Agranovich, J. Lumin. 2004, 110, 364 ; (b) P. Michetti, G. La Rocca, Phys. Rev. B 2009, 79, 035325 ; (c) L. Mazza, G. C. La Rocca, Phys. Rev. B 2009, 80, 235314.

[11] R. Anémian, J.-C. Mulatier, C. Andraud, O. Stéphan, J.-C. Vial, Chem. Commun. 2002, 3,1608 .

[12] J. Jo, C. Chi, S. Höger, G. Wegner, D. Y. Yoon, Chem. - A Eur. J. 2004, 10, 2681.

[13] C. Chi, C. Im, G. Wegner, J. Chem. Phys. 2006, 124, DOI 10.1063/1.2137709.

[14] S. Schumacher, A. Ruseckas, N. A. Montgomery, P. J. Skabara, A. L. Kanibolotsky, M. J. Paterson, I. Galbraith, G. A. Turnbull, I. D. W. Samuel, J. Chem. Phys. 2009, 131, 1.

[15] C. Chi, C. Im, G. Wegner, J. Chem. Phys. 2006, 124, 1.

[16] E. Y. Choi, L. Mazur, L. Mager, M. Gwon, D. Pitrat, J. C. Mulatier, C. Monnereau, A. Fort, A. J. Attias, K. Dorkenoo, J. E. Kwon, Y. Xiao, K. Matczyszyn, M. Samoc, D.-W. Kim, A. Nakao, B. Heinrich, D. Hashizume, M. Uchiyama, S. Y. Park, F. Mathevet, T. Aoyama, C. Andraud, J. W. Wu, A. Barsella, J. C. Ribierre, Phys. Chem. Chem. Phys. 2014, 16, 16941. 


\section{WILEY-VCH}

[17] (a) S. D. Lee, M. Sarmadi, F. Denes, J. L. Shohet, Plasmas Polym. 1997, 2, 177; (b) Z. Q. Hua, R. Sitaru, F. Denes, R. A. Young, Plasmas Polym. 1997, 2, 199; (c) H. Fujimoto, T. Miyayama, N. Sanada, C. Adachi, Org. Electron. physics, Mater. Appl. 2013, 14, 2994.

[18] J. Kasprzak, M. Richard, S. Kundermann, A. Baas, P. Jeambrun, J. M. J. Keeling, F. M. Marchetti, M. H. Szymańska, R. André, J. L. Staehli, V. Savona, P. B. Littlewood, B. Deveaud, L. S. Dang, Nature 2006, 443, 409.

[19] D. N. Krizhanovskii, K. G. Lagoudakis, M. Wouters, B. Pietka, R. A. Bradley, K. Guda, D. M. Whittaker, M. S. Skolnick, B. Deveaud-Plédran, M. Richard, R. André, L. S. Dang, Phys. Rev. B 2009, 80, 045317.

[20] D. M. Coles, P. Michetti, C. Clark, W. C. Tsoi, A. M. Adawi, J.-S. Kim, D. G. Lidzey, Adv. Funct. Mater. 2011, 21, 3691.

[21] (a) A. Baas, K. G. Lagoudakis, M. Richard, R. André, L. S. Dang, B. Deveaud-Plédran, Phys. Rev. Lett. 2008, 100, 2 ; (b) H. Ohadi, Y. Del Valle-Inclan Redondo, A. J. Ramsay, Z.

Hatzopoulos, T. C. H. Liew, P. R. Eastham, P. G. Savvidis, J. J. Baumberg, Phys. Rev. B 2018, $97,1$. 

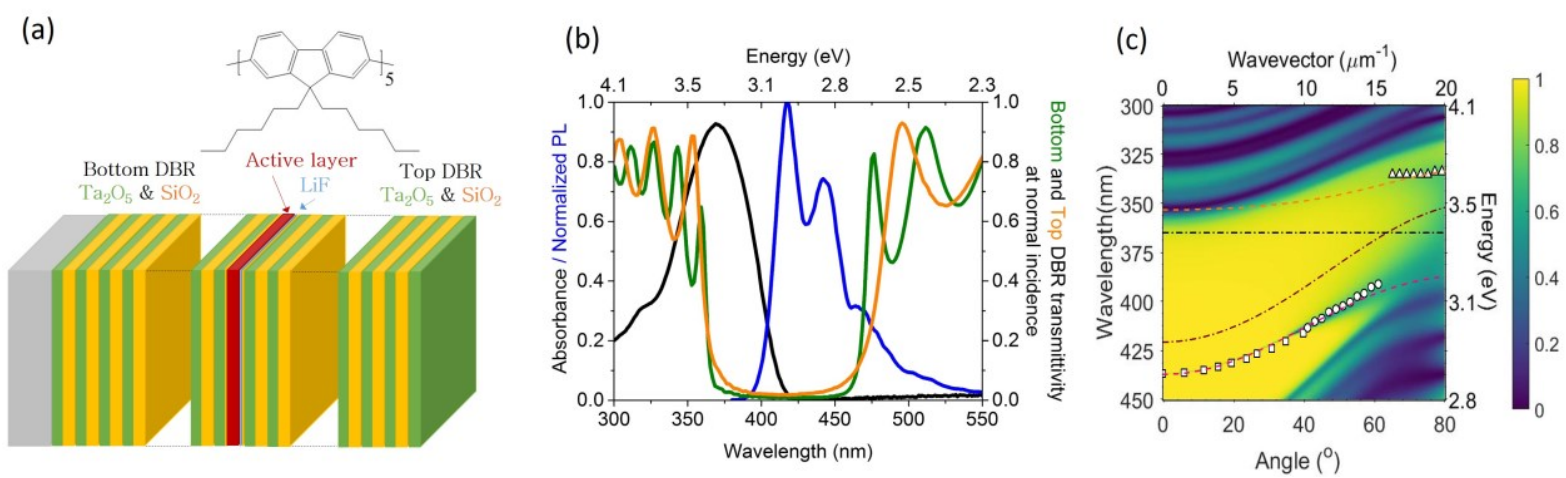

Figure 1: (a) Structure of pentafluorene, and sketch of the planar microcavity. (b) Absorption (black), steady state emission (blue) spectra of a neat pentafluorene film and transmission spectra through the sputtered bottom (green) and top (orange) reference DBRs at normal incidence. (c) Transfer matrix calculated spectra in pseudo color plots, the experimental reflectivity minima of the lower and upper polariton branch from cavity A as scatter plots and matching coupled oscillator model dispersion as dashed lines are depicted. 


\section{WILEY-VCH}
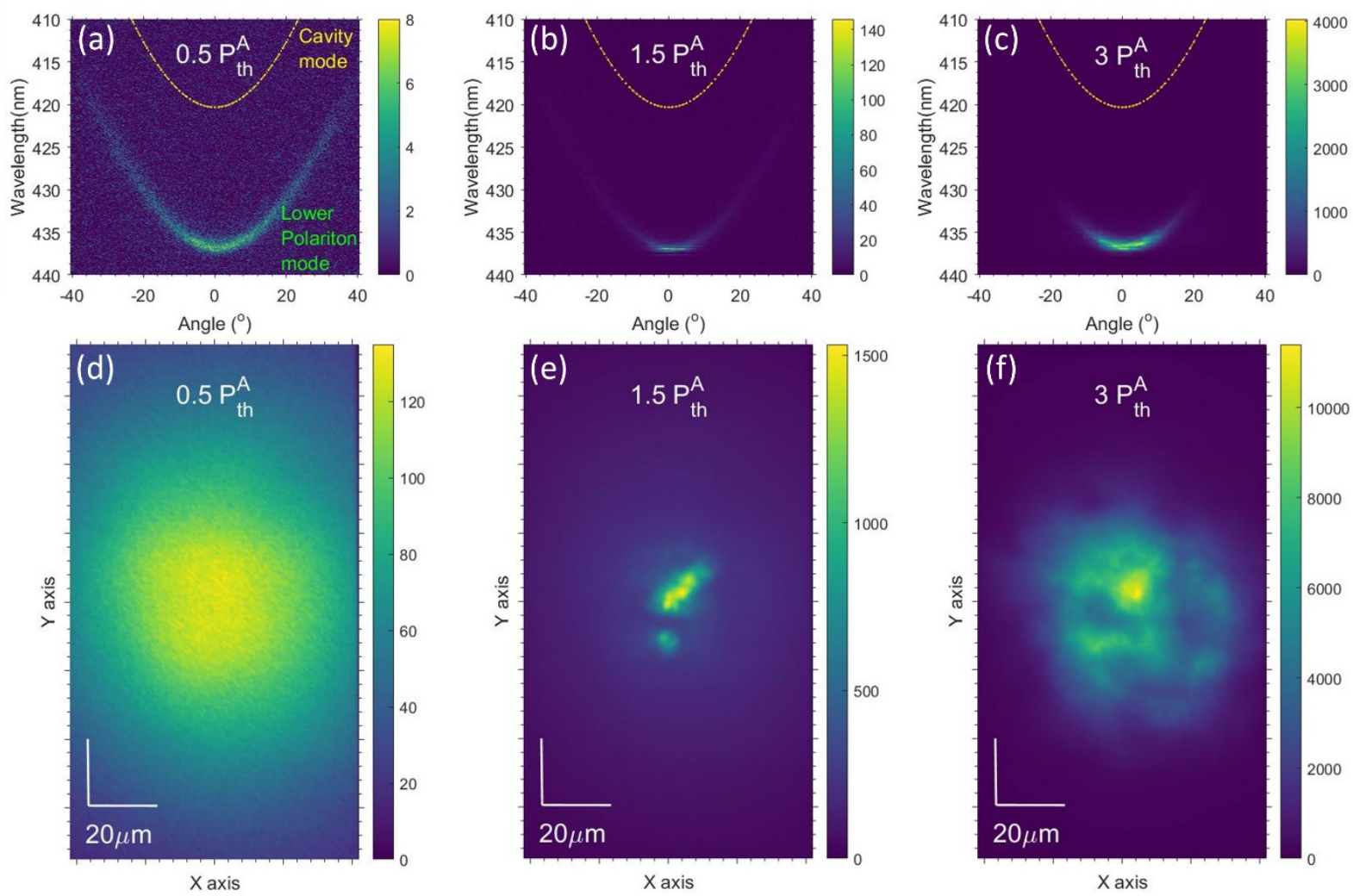

Figure 2: (a-c) Angle resolved lower polariton emission from Cavity A, with incident (absorbed) threshold excitation density $\left(P_{t h}^{A}\right)$ of $16.7 \mu \mathrm{J} \mathrm{cm}^{-2}\left(11.7 \mu \mathrm{J} \mathrm{cm}^{-2}\right)$ with estimated cavity mode as dotted lines. The emission dispersion below threshold (a), above threshold (b), and at $3 P_{t h}^{A}$ (c). Images (d-f) show corresponding real space distribution of emission. 


\section{WILEY-VCH}
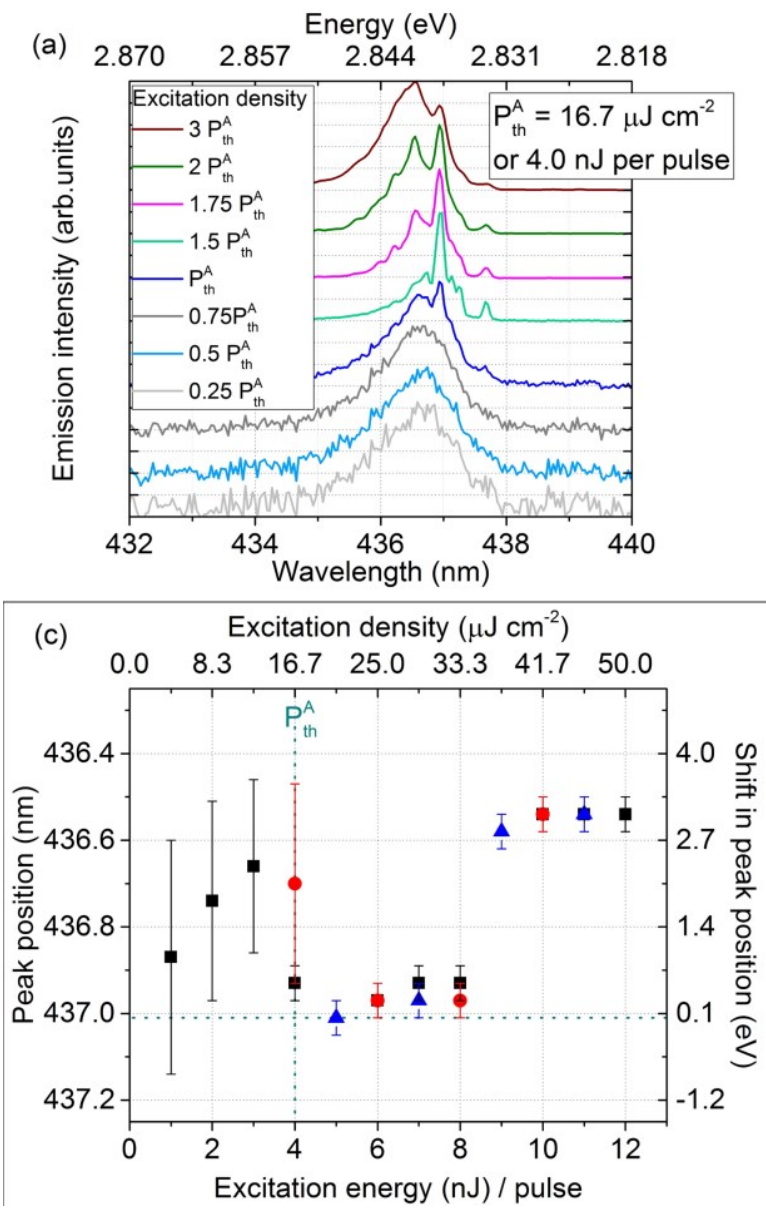
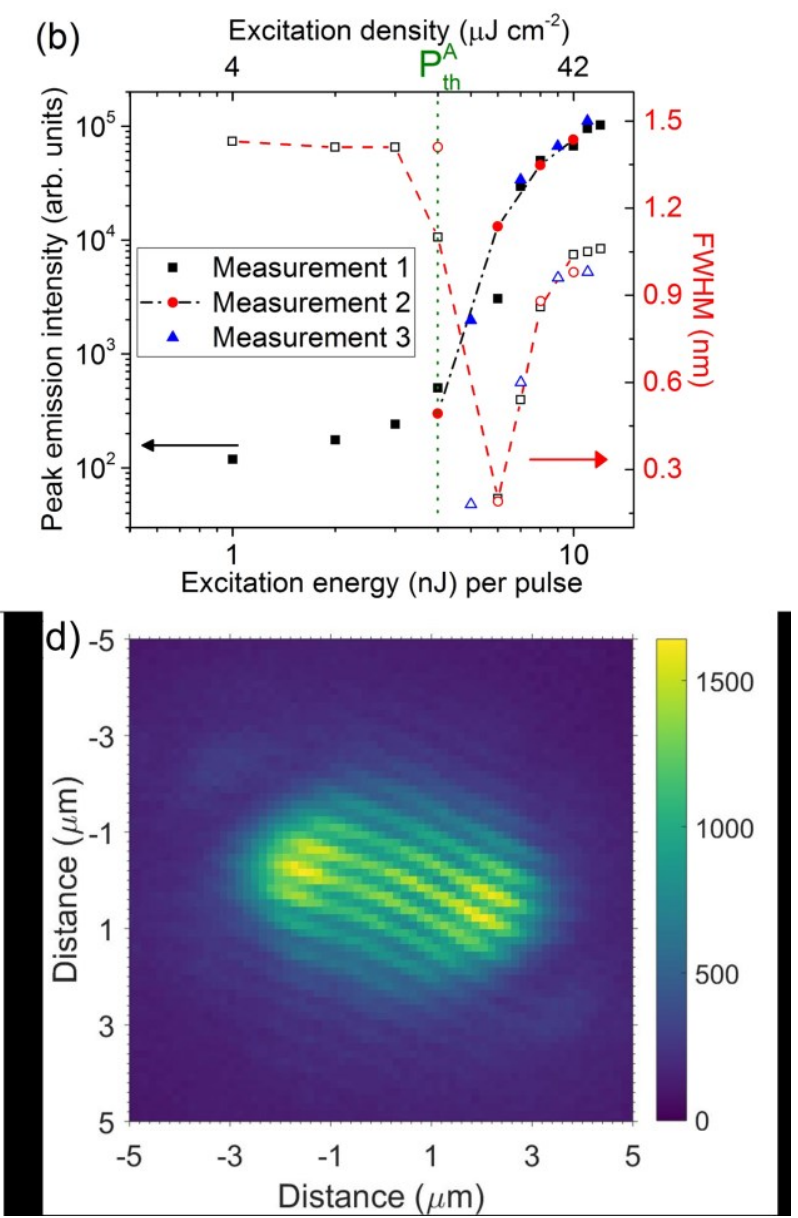

Figure 3: (a) Normalized and vertically translated emission spectra from cavity A collected in the range of $\pm 5^{\circ}$ with change in excitation density. (b) Peak emission intensity (solid symbols) from cavity A shows non-linearity with excitation density and drop in spectral FWHM (hollow symbols) at threshold. (c) Peak emission wavelength from cavity shows an initial red shift at threshold followed by a blue shift with increasing polariton density. (d) An interferogram at $26 \mu \mathrm{J} \mathrm{cm}^{-2}$ incident excitation density showing long-range spatial coherence in cavity A. 
Table I: Summary of room temperature microcavity polariton lasing thresholds from strongly coupled organic and inorganic semiconductor materials

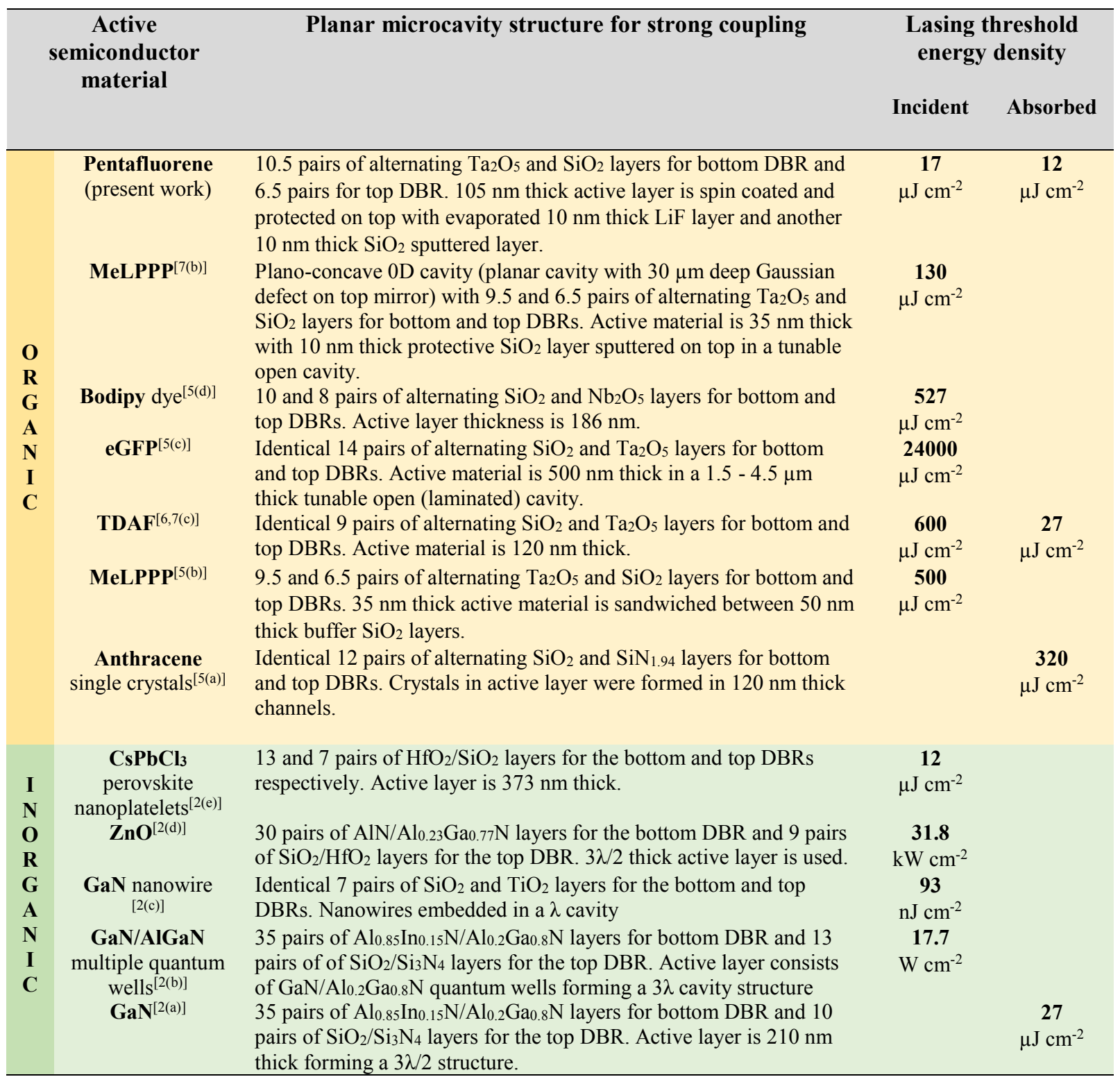




\section{WILEY-VCH}

Strong light-matter coupling is a promising route towards coherent light sources because it has the potential for thresholdless polariton lasing. Here we demonstrate that pentafluorene is a very good material for strong coupling and low threshold polariton lasing. We obtain a low threshold of $17 \mu \mathrm{J} \mathrm{cm}^{-2}$ by making use of optimum active material and fabrication techniques. This study will assist the development of low threshold electrically pumped lasing from polariton devices.

Keyword: polariton lasing

Sai Kiran Rajendran, Mengjie Wei, Hamid Ohadi, Arvydas Ruseckas, Graham A. Turnbull*, Ifor D. W. Samuel*

Low threshold polariton lasing from a solution processed organic semiconductor in a planar microcavity

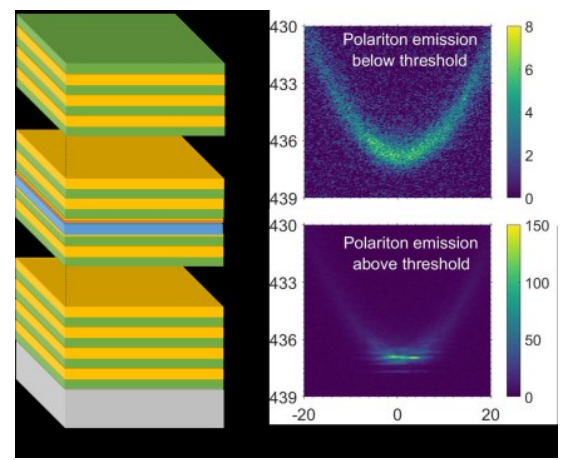




\section{WILEY-VCH}

Copyright WILEY-VCH Verlag GmbH \& Co. KGaA, 69469 Weinheim, Germany, 2016.

\section{Supporting Information}

\section{Low threshold polariton lasing from a solution processed organic semiconductor in a planar microcavity}

Sai Kiran Rajendran, Mengjie Wei, Hamid Ohadi, Arvydas Ruseckas, Graham A. Turnbull*, Ifor D. W. Samuel*

\section{Section 1: Effect of protective lithium fluoride layer}

It is well known that sputter deposition on an organic semiconductor layer can result in damage to the layer. Though studies regarding sputter deposition of top cathode in OLEDs have reported better performance compared to vacuum heat-deposited contacts ${ }^{[1]}$ further improvements to OLED performance were made by including a buffer layer to reduce plasma damage ${ }^{[2,3]}$ and penetration of ions into the organic layer ${ }^{[4]}$ that can occur during sputtering process. In our study we decided to evaporate a transparent $10 \mathrm{~nm}$ thick lithium fluoride layer as a buffer on top of the pentafluorene film to reduce plasma damage. The photoluminescence quantum yield (PLQY) from pentafluorene films exposed to different processes are depicted in figure S1. The PLQY from the film deposited on quartz substrate was recorded with both $343 \mathrm{~nm}$ as well as $370 \mathrm{~nm}$ excitation, depicted as solid and empty scatter plots respectively in figure S1(a). While the PLQY with different excitation wavelengths are different they follow the same pattern. Exposure of the film to vacuum and heat in evaporation chamber to deposit $10 \mathrm{~nm}$ lithium fluoride on top reduced the PLQY. Further exposure to argon/oxygen plasma during sputter deposition of $10 \mathrm{~nm}$ thick $\mathrm{SiO}_{2}$ or $47 \mathrm{~nm}$ thick $\mathrm{Ta}_{2} \mathrm{O}_{5}$ reduced the PLQY even further. However, exposure to plasma without the protective lithium fluoride layer drastically reduces the PLQY of pentafluorene from $90 \%$ to $35 \%$. The subsequent deposition of $47 \mathrm{~nm}$ thick $\mathrm{Ta}_{2} \mathrm{O}_{5}$ on $10 \mathrm{~nm}$ thick $\mathrm{SiO}_{2}$ results in an increase in the PLQY. This could be due to onset of cavity enhanced emission. Similar measurements from pentafluorene films on glass 
substrates with $370 \mathrm{~nm}$ excitation, shown in figure $\mathrm{S} 1(\mathrm{~b})$, reaffirm the protective action of the lithium fluoride layer.
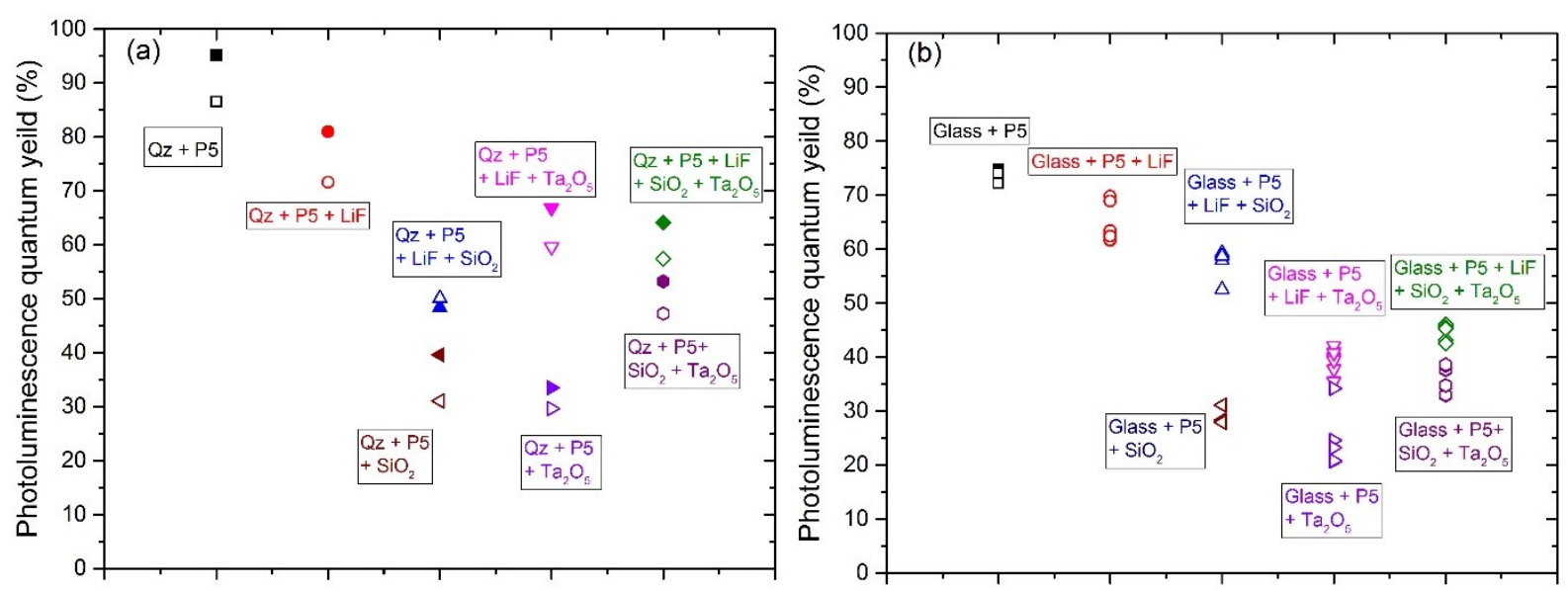

Figure S1: (a) Photoluminescence quantum yield recorded from pentafluorene (P5) films spin coated on quartz $(\mathrm{Qz})$ substrates with excitation wavelengths of $343 \mathrm{~nm}$ (solid symbols) or $370 \mathrm{~nm}$ (hollow symbols) using a Hamamatsu Photonics absolute quantum yield spectrometer.

(b) Similar measurement from pentafluorene films on glass substrates with $370 \mathrm{~nm}$ excitation show the same trend.

\section{Section 2: Reflectivity measurements on cavities}

Reflectivity spectra measured from the three cavities A, B and C show the peak position of the polariton modes with angle tuning. At normal incidence, while the lower polariton mode is clearly visible in the three negatively detuned cavities, the upper polariton mode is outside the reflectivity stop band of the mirrors. At high angles of incidence, the lower polariton mode shifts to higher energies at a much slower rate than the DBR band gap edge as a result of strong coupling. This results in the lower polariton mode being enveloped by the DBR band edge. However, this also allows the upper polariton mode to emerge within the DBR band gap at high angles. The large Rabi splitting energy and short band gap of the DBRs fabricated prevent simultaneous observation of both the polariton modes at a wide range of 


\section{WILEY-VCH}

angles. From the dispersion of the modes, spectra shown in Figure S2, it is evidenced that the cavities are strongly coupled

For angles shorter than $40.5^{\circ}$ (limited by the numerical aperture of the objective lens), reflected and emission spectra were collected using Fourier plane imaging. Figure S3 shows that reflectivity and photoluminescence spectra from the three cavities show the same dispersion relation. The polariton dispersion for cavity A from figures S2 and S3 are used in Figure 3(c) in the main text.

(a)

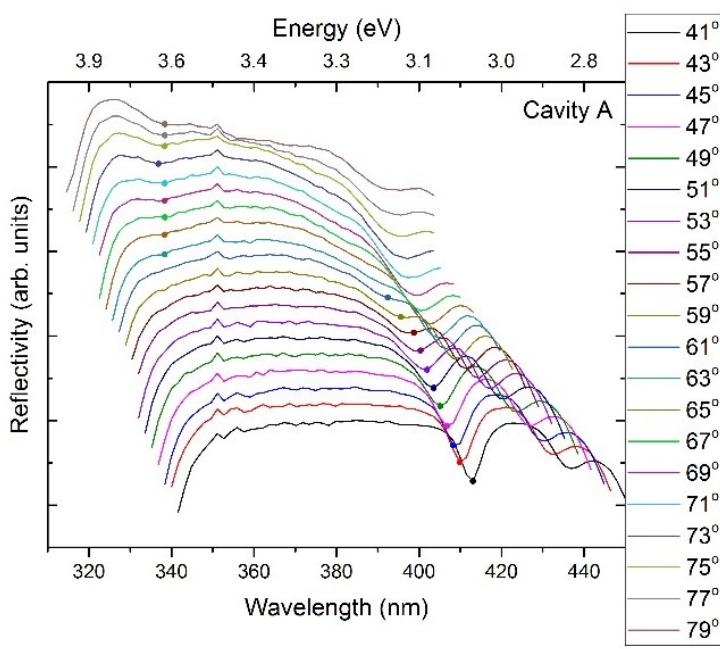

(c)

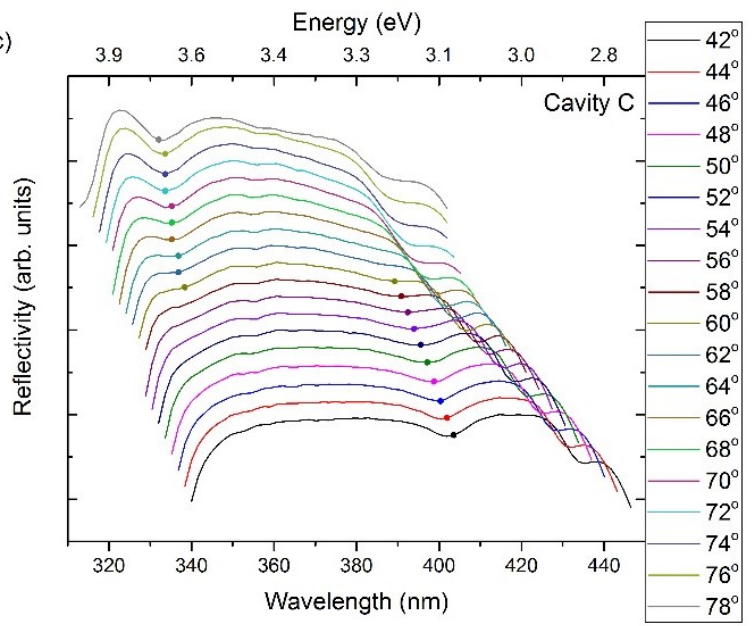

(b)

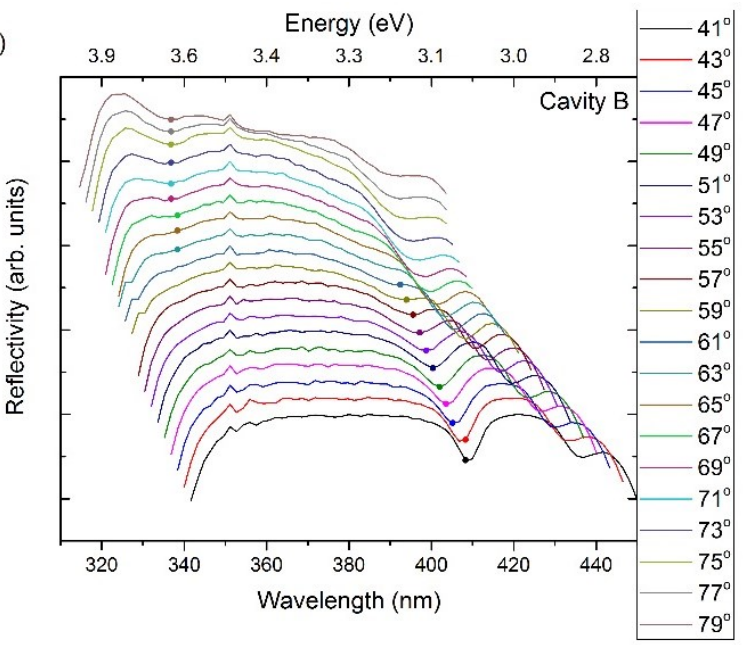

Figure S2: Normalized experimental reflectivity spectra, vertically translated for improved visibility, show position of the lower and upper polariton modes with angle tuning for the three cavities A, B and C (a-c). The peak positions in the spectra are highlighted with scatter plots. 


\section{WILEY-VCH}
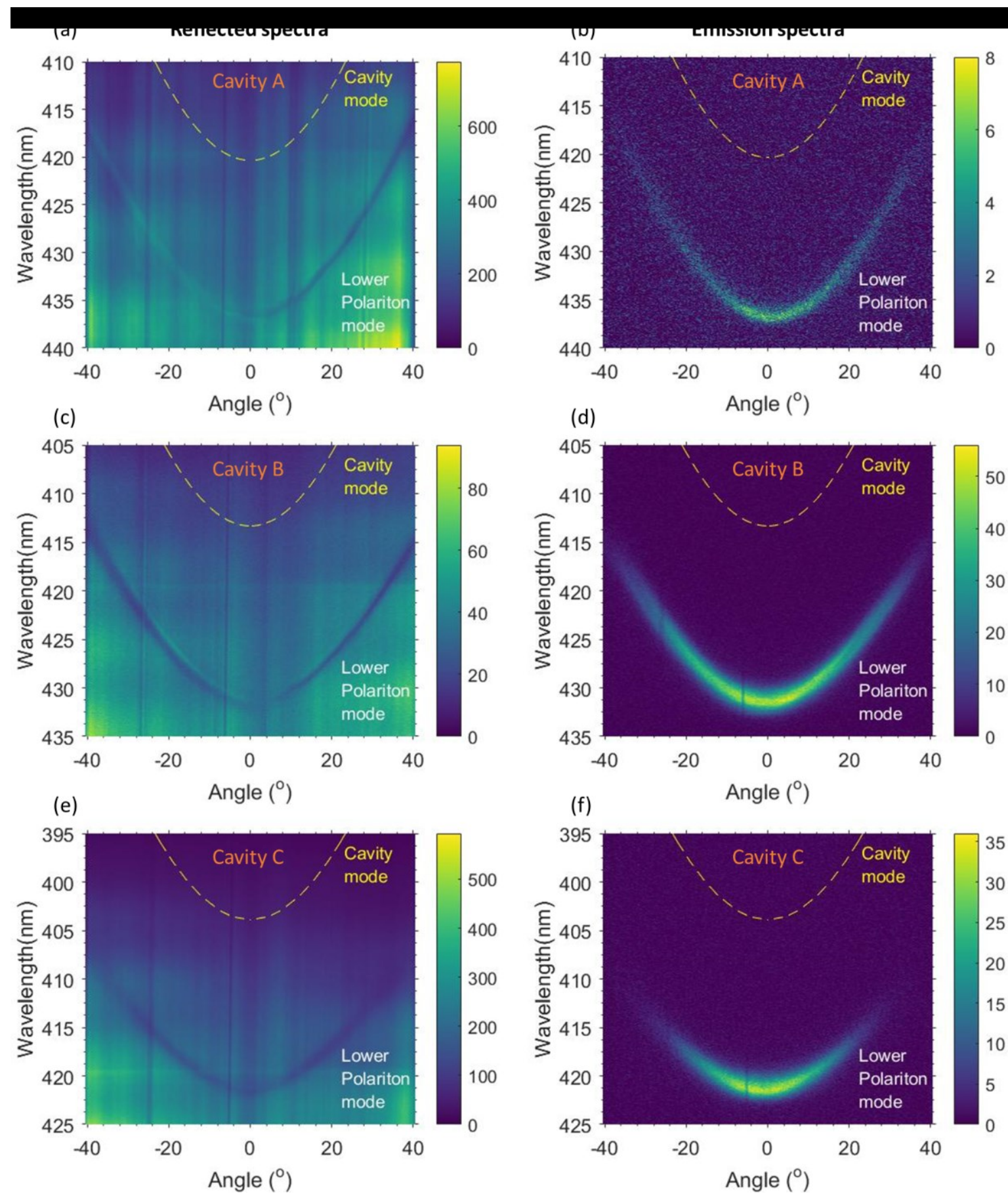

Figure S3: The xenon lamp reflected spectra from the three cavities A, B and C are shown in

figures (a, c, e respectively) while the emission spectra from the same spot from the corresponding cavities are shown in figures $(b, d, f)$. The reflected and emission dispersion observed are from the same lower polariton mode. The cavity modes of the three cavities obtained from coupled oscillator model are at much shorter wavelengths than the lower polariton modes. 


\section{WILEY-VCH}

\section{Section 3: Ellipsometric measurement on pentafluorene films}

Spin coated small molecules in general are largely isotropic. ${ }^{[5]}$ It has also been reported that pentafluorene in neat films are only slightly oriented along the substrate plane ${ }^{[6]}$ Figure S4 shows the refractive index fits for pentafluorene obtained using variable angle spectroscopic ellipsometry performed on pentafluorene films on silicon substrates. The thickness of the film was first extracted using a Cauchy model followed by a refractive index fit for the entire region of interest using multiple regression using Woollam WVASE software for an isotropic model. This complex refractive index was then used to calculate the transmission/reflection through the strongly coupled microcavity using transfer matrix method.

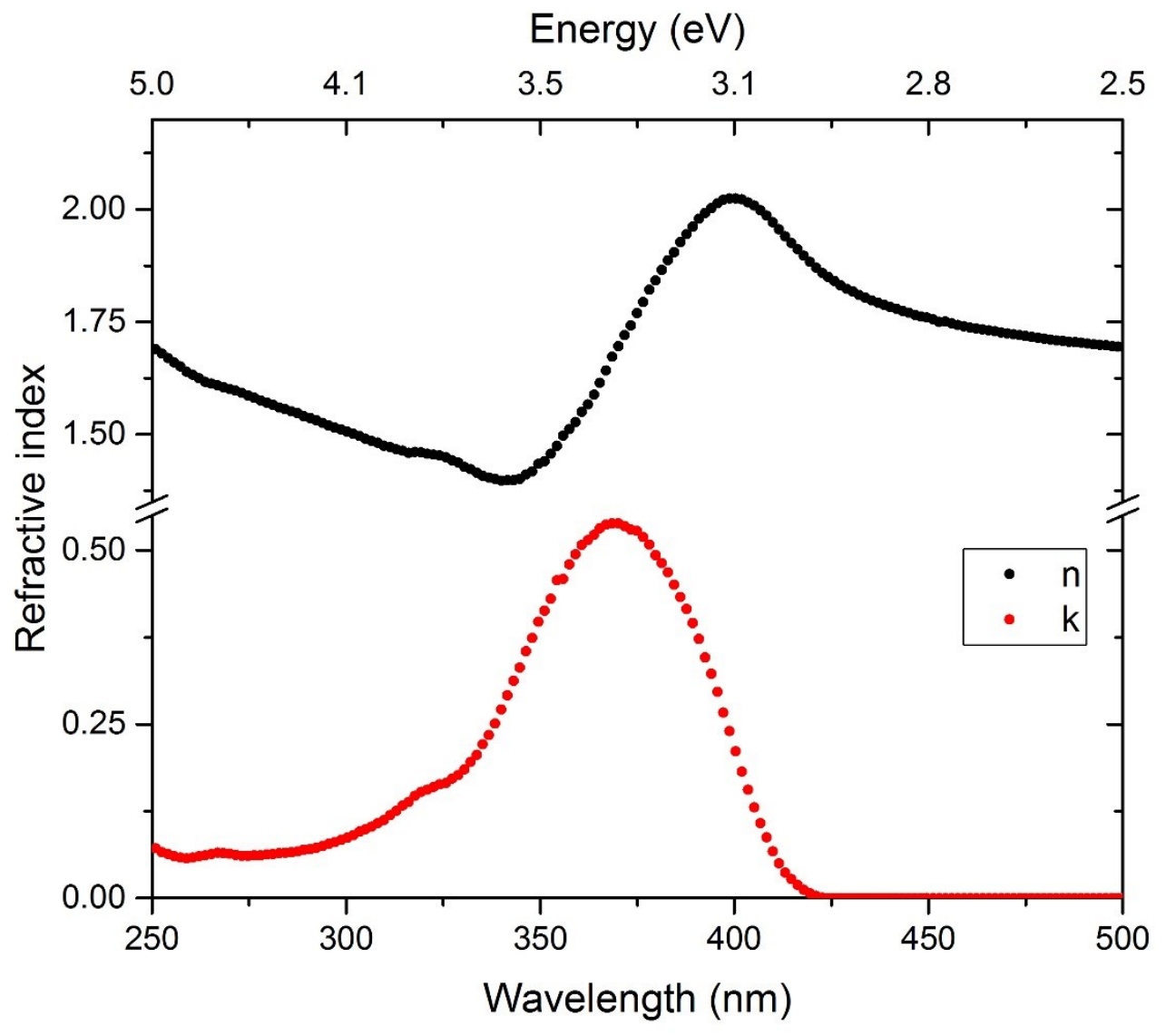

Figure S4: Real (n) and imaginary (k) parts of the refractive index fit of a pentafluorene film 


\section{WILEY-VCH}

\section{Section 4: Coupled oscillator model}

The photonic $\left(E_{p}\right)$ and exciton $\left(E_{e x}\right)$ modes can be represented as oscillators ${ }^{[7,8]}$ whose interaction strength is represented by the parameter $\Omega$. This gives an eigenvalue equation for the energies of the coupled oscillators, written as $\left[\begin{array}{cc}E_{p} & \Omega \\ \Omega & E_{\theta x}\end{array}\right]\left[\begin{array}{l}\alpha \\ \beta\end{array}\right]=E_{U, L}\left[\begin{array}{l}\alpha \\ \beta\end{array}\right]$. The eigenvalues are $E_{U, L}=1 / 2\left(\left(E_{p}+E_{\theta x}\right) \pm \sqrt{\left(E_{p}-E_{e x}\right)^{2}+4 \Omega^{2}}\right)$, show the dispersion of the upper and lower polarition modes. $\alpha^{2}$ and $\beta^{2}$ are the photon and exciton fractions of the lower/upper polariton modes. The experimental dispersion observed in the three cavities A, B \& C are shown as scatter plots in figure S5 (a-c). A small step in the dispersion around $42^{\circ}$ angle of incidence is due to difference between the two experimental setup used to measure the reflectivity at small and large angles. The input variables, cavity mode energy at normal incidence, exciton mode energy from peak absorption, the effective refracitive index of the cavity and the Rabi splitting energy into the coupled oscillator equation, shown in table ST1, give a dispersion (solid line in figures S5 (a-c)) that agree well the experimental results. The Rabi splitting energies from the three negatively detuned cavities are nearly identical at 500 $\mathrm{meV}$. The photon/exciton fractions or the Hopfield coefficients, shown in figure S5 (d-f) for the three cavities show that photonic fraction of the lower polariton at normal incidence is nearly $80 \%$. 

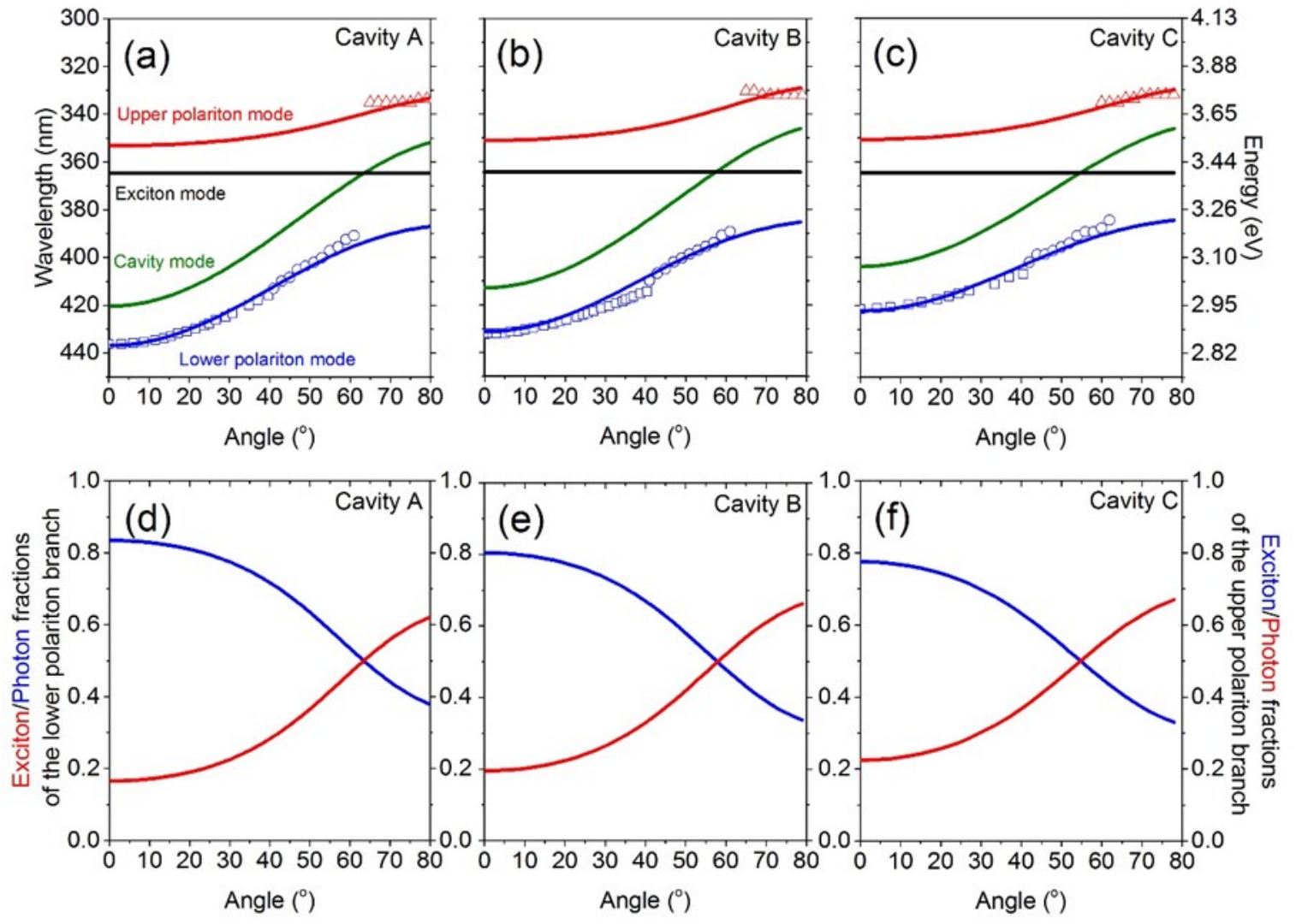

Figure S5: Polariton peak positions as scatter plots and coupled oscillator model dispersion as solid lines for cavities A, B and C (a-c) and Hopfield coefficients for corresponding cavities (d-f).

Table ST1: Inputs and parameters used in coupled oscillator model to match with experimental data

\begin{tabular}{|c|c|c|c|c|c|c|c|}
\hline $\begin{array}{c}\text { Cavity } \\
\text { name }\end{array}$ & $\begin{array}{c}\text { Lower } \\
\text { polariton at } \\
\text { normal } \\
\text { incidence } \\
(\mathrm{nm} ; \mathrm{eV})\end{array}$ & $\begin{array}{c}\text { Cavity mode } \\
\text { at normal } \\
\text { incidence } \\
(\mathrm{nm} \& \mathrm{eV})\end{array}$ & $\begin{array}{c}\text { Exciton } \\
\text { mode } \\
\text { peak } \\
(\mathrm{eV})\end{array}$ & $\begin{array}{c}\text { Negative } \\
\text { detuning } \\
(\mathrm{meV})\end{array}$ & $\begin{array}{l}\text { Effective } \\
\text { refractive } \\
\text { index }\end{array}$ & $\begin{array}{c}\text { Half-Rabi } \\
\text { splitting } \\
\text { energy } \\
\Omega(\mathrm{meV})\end{array}$ & 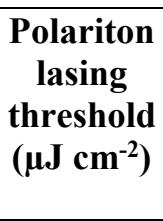 \\
\hline A & $436.4 ; 2.84$ & $420.3 ; 2.95$ & 3.4 & 450 & 1.8 & 250 & 16.6 \\
\hline B & $432.6 ; 2.86$ & $413.3 ; 3.0$ & 3.4 & 400 & 1.8 & 260 & 20 \\
\hline $\mathrm{C}$ & $421.6 ; 2.94$ & $404 ; 3.07$ & 3.4 & 330 & 1.9 & 250 & 46 \\
\hline
\end{tabular}




\section{WILEY-VCH}

\section{Section 5: Lasing studies on differently detuned cavities}

In order to understand the population and relaxation dynamics of the lower polariton branch, cavities of different detunings were studied. If radiative decay is the dominant mechanism, then the relaxation rate is similar to the radiative decay rate. With increasing excitation density, the polaritons at the bottom of the lower polariton branch decay quickly due to their higher photonic fraction. When this emission overlaps with the gain spectrum of the material, population into the bottom of lower polariton branch is quickly enhanced. This results in lowering the threshold for the onset of polariton lasing in materials with high PLQY. As seen in our cavities, A and B have a threshold lower than cavity C. However, the nature of lasing from all the cavities are nearly the same. Shown here in figure S6, are the Fourier and real space emission from cavity B (S6, a-f) and cavity C (S6, g-1). Below threshold excitation densities, both the cavities show emission following lower polariton dispersion (S6, a and g) in Fourier space, while the real space emission (S6, d and j) follows the Gaussian excitation profile. Above threshold excitation density, multiple narrow spectra emerge in Fourier space (S6, b and h) arising from localized spots (S6, e and k) are observed. Increasing excitation density further increases the polariton density of the bright spots resulting in a blue shifted emission (S6, c and i) in Fourier space and a corresponding spatial spread of distribution in real space (S6, f and 1) due to repulsive interaction. Slide show videos of the recorded emission from the three cavities in Fourier space are available with this supporting document. The increase and decrease with emission intensity and the change in polariton emission dispersion as the excitation density in varied is depicted.

The multiple narrow polariton lasing modes observed from cavities B and C are depicted in figure S7 (a) and (b) respectively as normalized and vertically translated spectra. It can be seen that while there is a shift in emission to the blue, it is due to relative increase peak intensities rather than movement of the peaks themselves. The non-linear increase in emitted 


\section{WILEY-VCH}

intensity as well as drop in spectral full width at half maximum from the two cavities are shown in figure S7 (c) and (d) respectively.
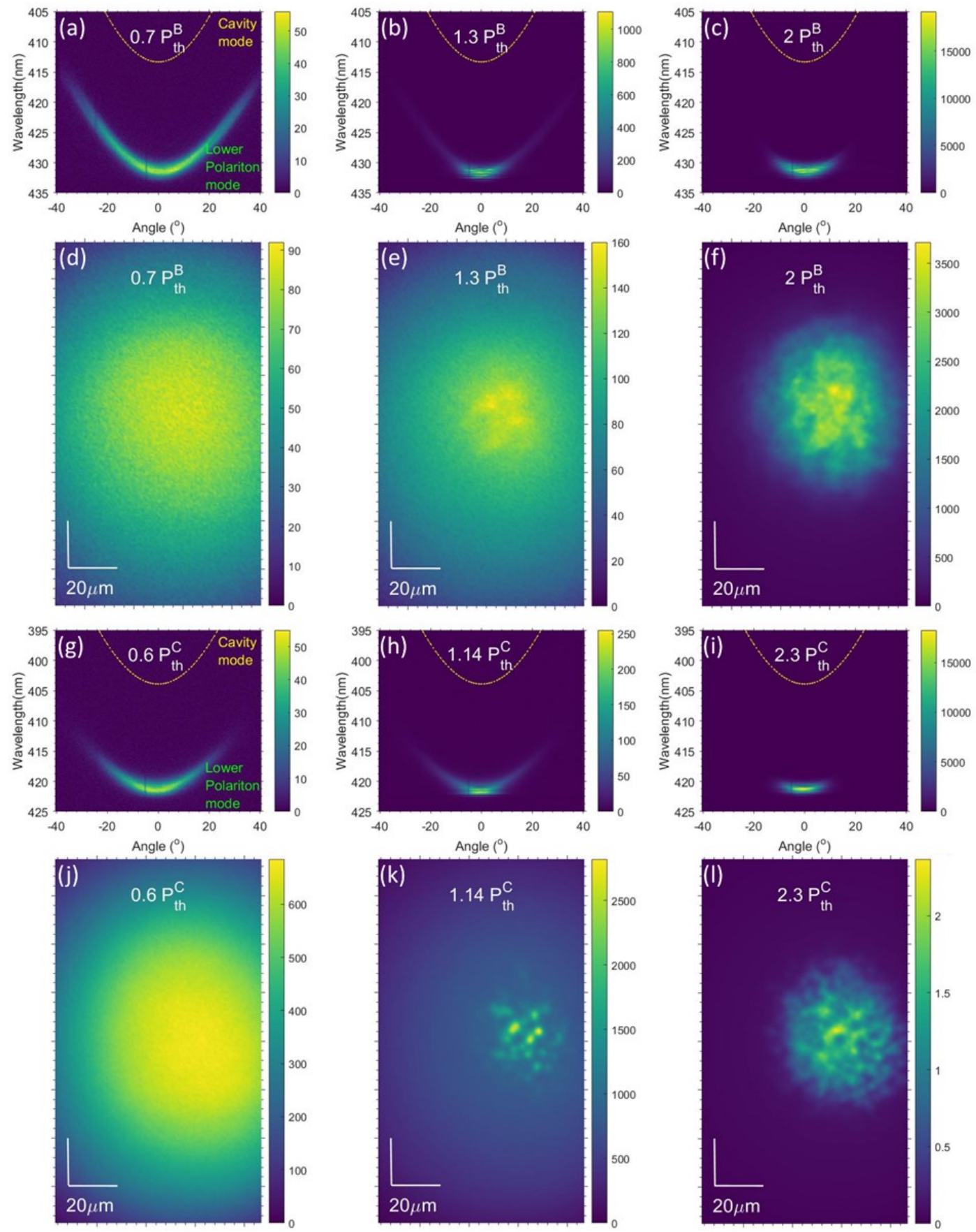

Figure S6: Fourier space emission from cavity B at three different excitation densities, $0.7 P_{t h}^{B}$ (a), $1.3 P_{t h}^{B}(\mathrm{~b})$, and $2.5 P_{t h}^{B}$ and their corresponding real space emission (d-f) respectively.

Similar emission from cavity $\mathrm{C}$, at three different excitation densities, $0.5 P_{t h}^{C}(\mathrm{~g}),{ }_{t h}^{C}(\mathrm{~h})$, and $1.5_{t h}^{C}$ in Fourier space and corresponding emission in real space $(\mathrm{j}-1)$ respectively. 
(a)

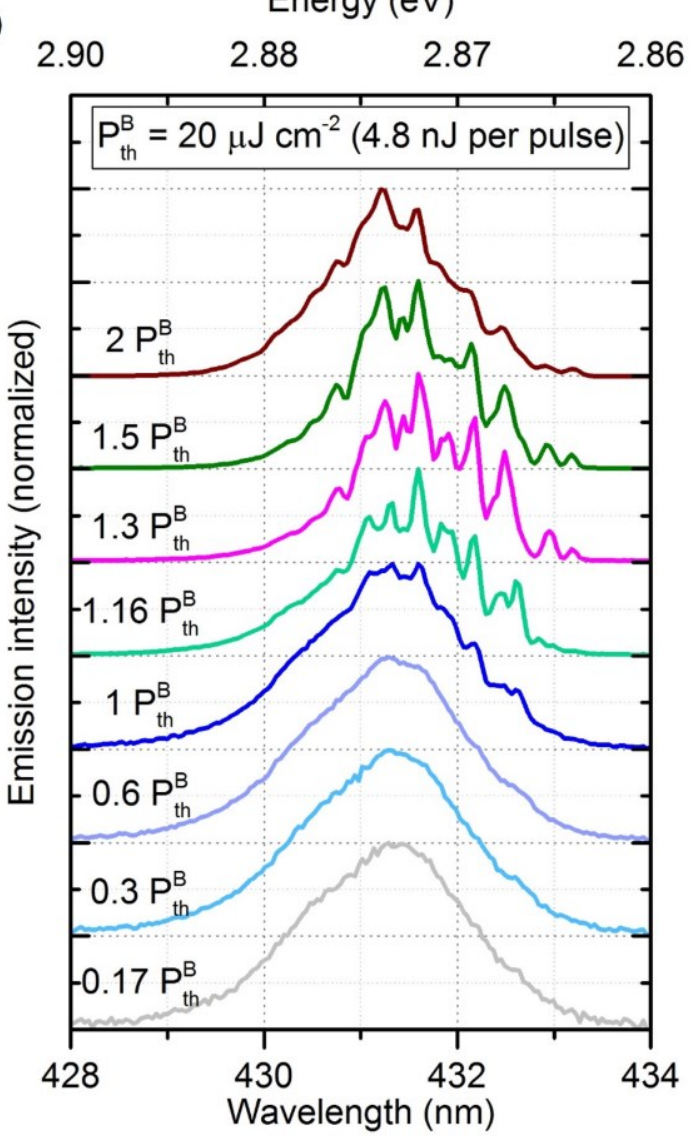

(c) Excitation density $\left(\mu \mathrm{J} \mathrm{cm}^{-2}\right)$

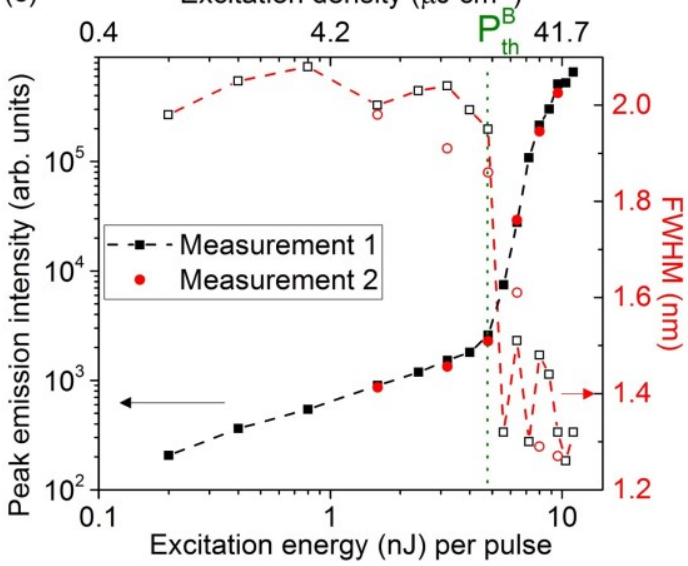

(b)

$\begin{array}{llll}2.97 & 2.95 & 2.94 & 2.92\end{array}$
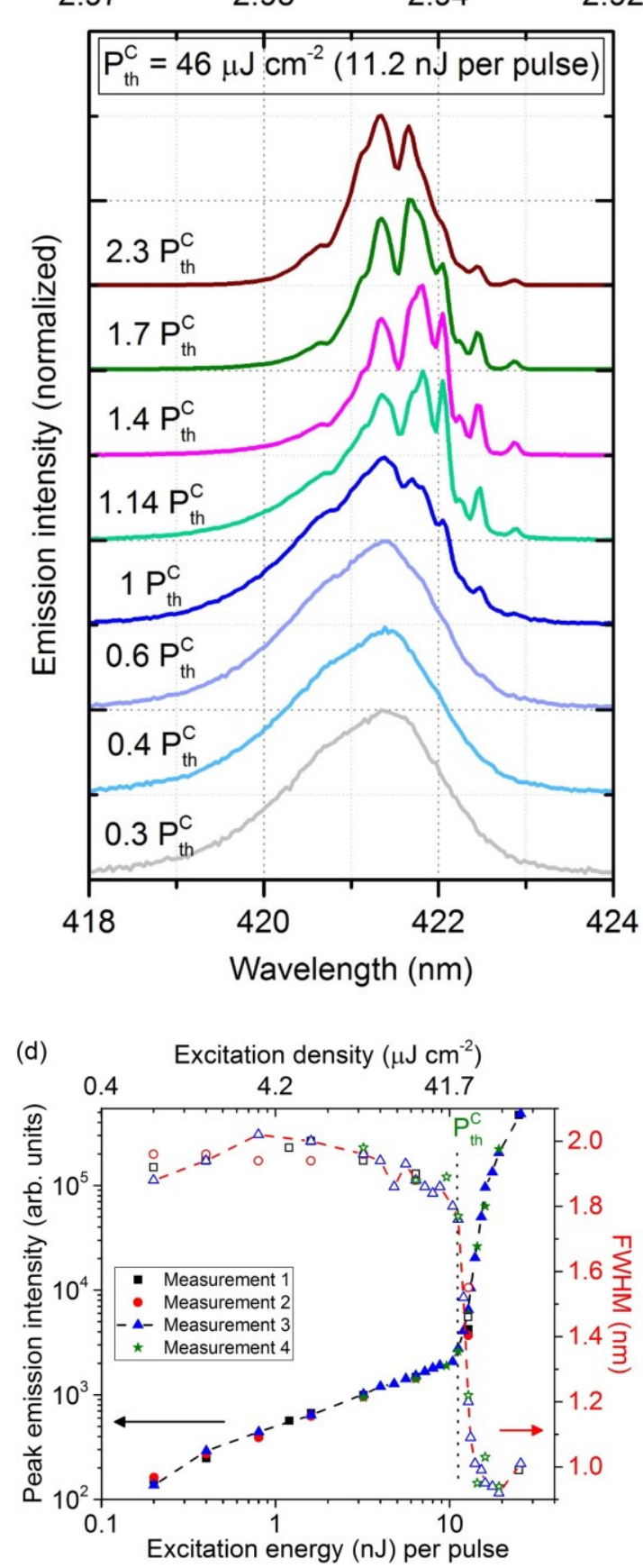

Figure S7: Normalized and vertically translated emission spectra from the cavities B and C with different excitation densities are shown in (a) and (b) respectively. (c-d) Peak emitted intensity collected from corresponding cavities collected in the range of $\pm 5^{\circ}$ show threshold behavior at incident excitation densities of $P_{t h}^{B}=20 \mu \mathrm{J} \mathrm{cm}^{-2}$ and $P_{t h}^{C}=46 \mu \mathrm{J} \mathrm{cm}^{-2}$ respectively. A drop in spectral FWHM at threshold is observed. 


\section{WILEY-VCH}

\section{Section 6: Polarization measurements on polariton emission}

The linear polarization of emission from the lower polariton branch was analyzed for two different linear polarizations of the excitation pump pulse. It is observed that below threshold excitation density, the emission is largely unpolarized while above threshold excitation density, the emission followed the pump polarization.
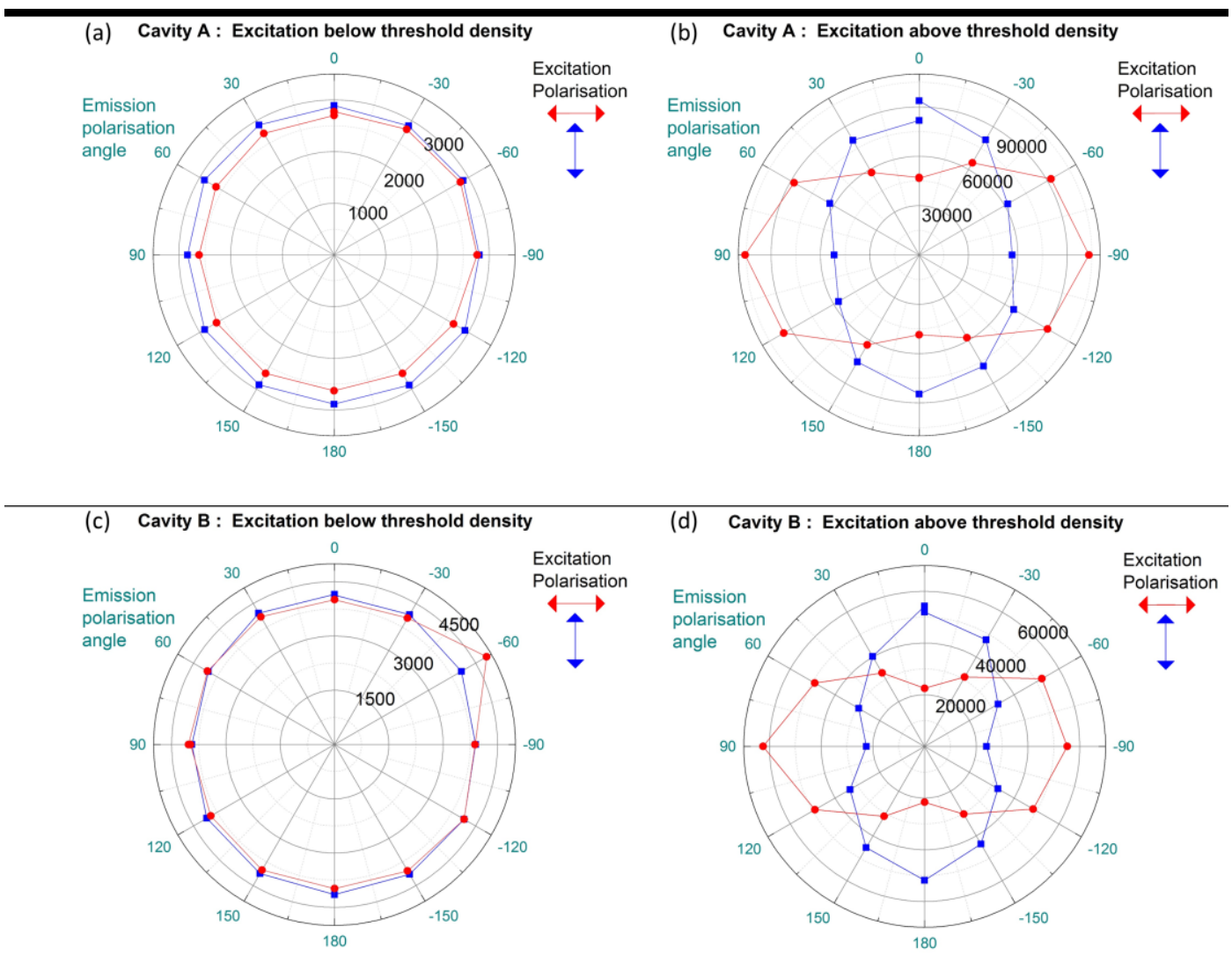

(e) Cavity C : Excitation below threshold density

(f) Cavity C : Excitation above threshold density
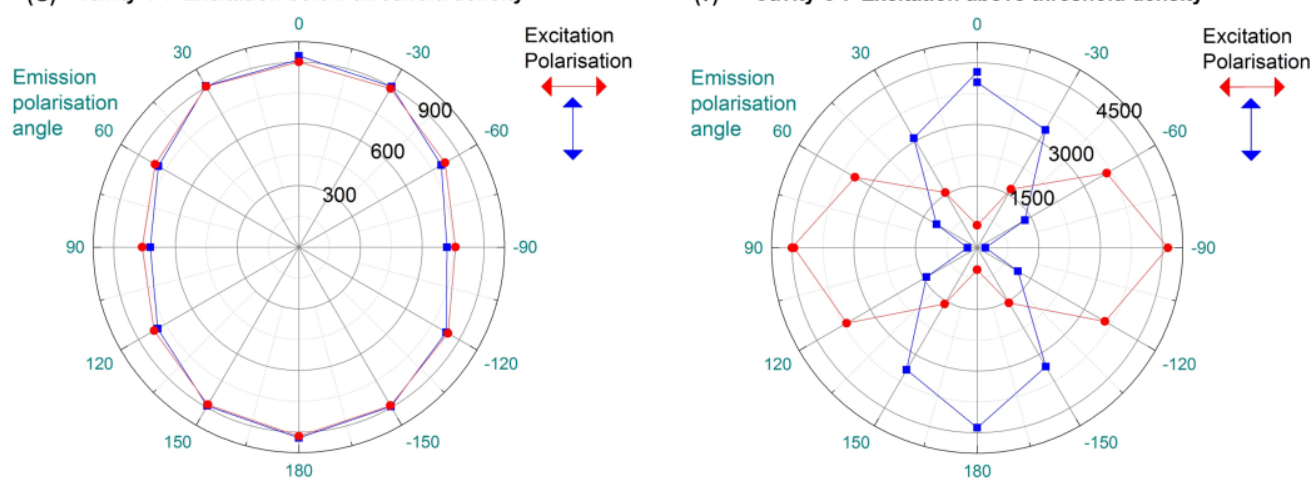

Figure S8: Emission intensities in polar plots for different angles of linear polarization for two different excitation linear polarization are shown with excitation densities below (a, c, e) and above (b, d, f) threshold excitation densities from cavities A, B and C. 


\section{WILEY-VCH}

\section{Section 7: Coherence measurements on the polariton lasers}

Additional videos of coherence measurements are available with this supporting document.
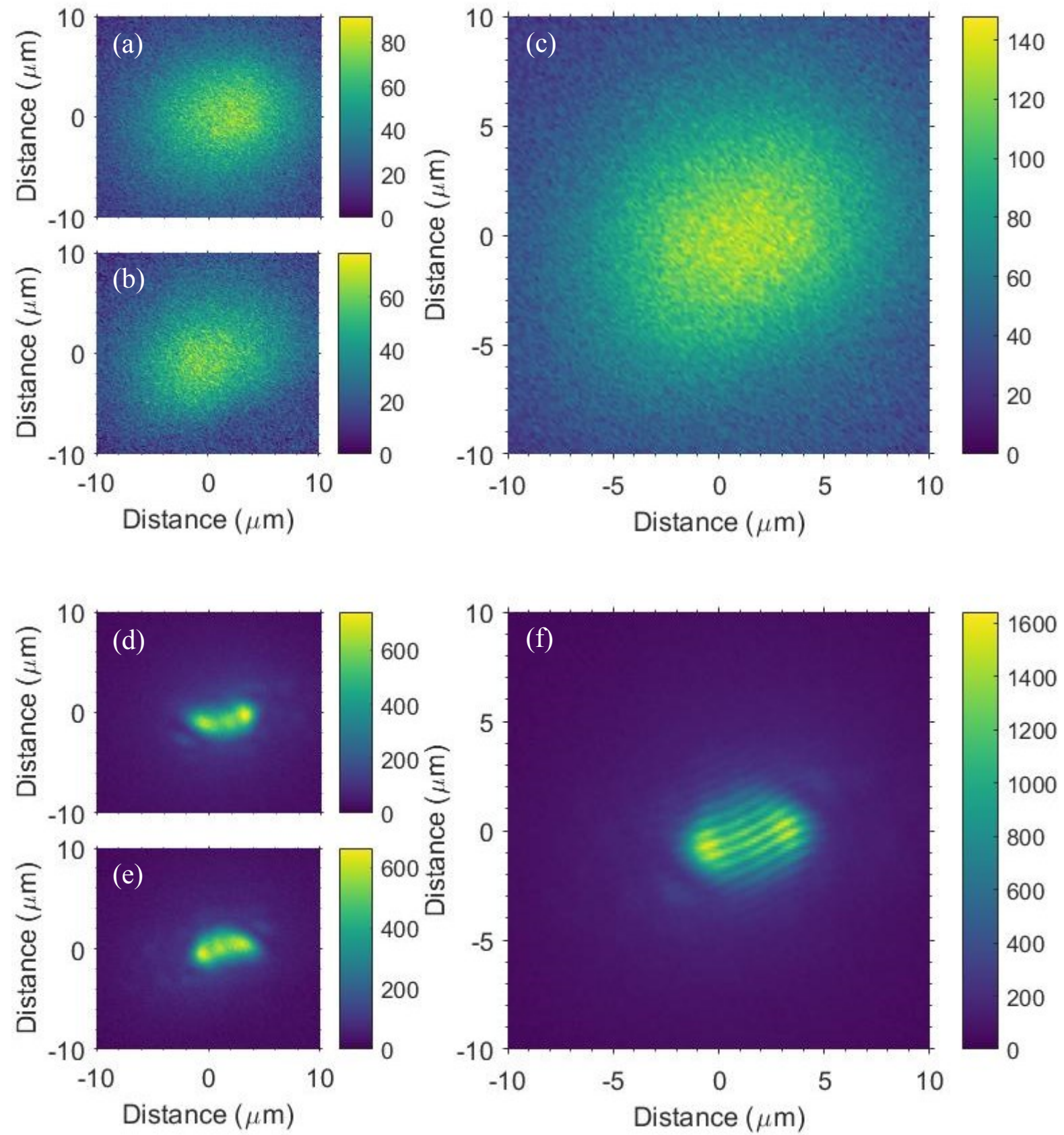

Figure S9: Images of the emitting spot collected through both arms of an interferometer (on the left) and the image of emitting spot from the two arms overlapped spatially and temporally (on the right) show absence of fringes below threshold, at $0.6{ }^{P^{A^{\prime}}}$ (top), and presence of fringes above threshold, at $1.3 P_{t h}^{A^{\prime}}$ (bottom), from Cavity A where $P_{t h}^{A^{\prime}}$ is incident $20 \mu \mathrm{J} \mathrm{cm}{ }^{-2}$. 


\section{WILEY-VCH}
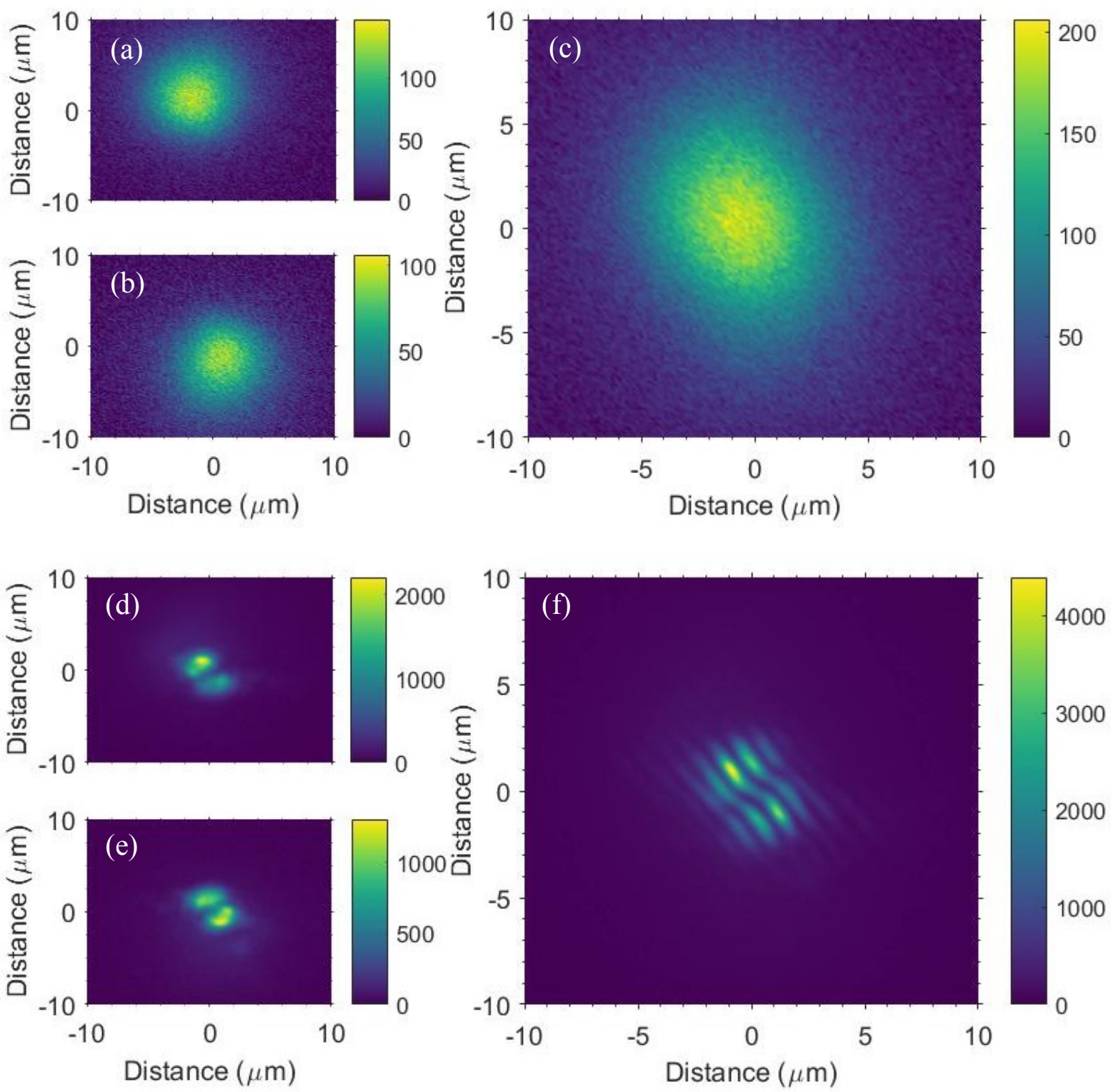

Figure S10: Images of the emitting spot collected through both arms of an interferometer (on the left) and the image of emitting spot from the two arms overlapped spatially and temporally (on the right) show absence of fringes below threshold, at $0.6{ }^{P^{B^{\prime}}}$ (top), and presence of fringes above threshold, at $1.16{ }^{P_{t h}^{B^{\prime}}}$ (bottom), from Cavity B where $P_{t h}^{B^{\prime}}$ is incident $32 \mu \mathrm{J} \mathrm{cm}{ }^{-2}$. 


\section{WILEY-VCH}
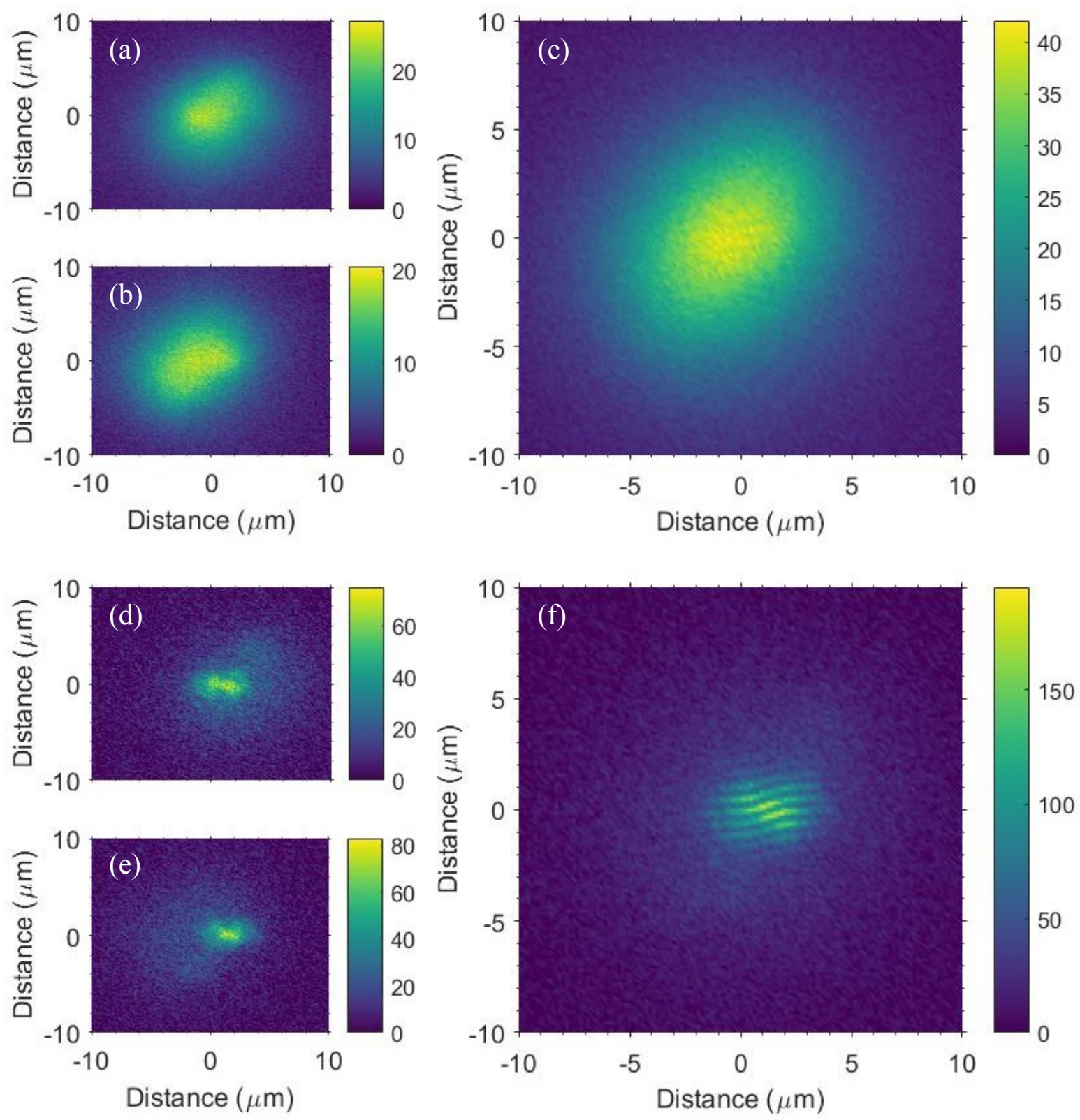

Figure S11: Images of the emitting spot collected through both arms of an interferometer (on the left) and the image of emitting spot from the two arms overlapped spatially and temporally (on the right) show absence of fringes below threshold at $0.8 P_{t h}^{C^{\prime}}$ (top) and presence of fringes above threshold at $1.4 P_{t h}^{C^{\prime}}$ (bottom) from Cavity $\mathrm{C}$ where $P_{\text {th }}^{C^{\prime}}$ is incident $68 \mu \mathrm{J} \mathrm{cm}$. 


\section{WILEY-VCH}

\section{Section 8: Pentafluorene film emission lifetime}

The time resolved emission from a neat pentafluorene film was measured using a Hamamatsu C10910 Streak camera connected to a spectrograph in synchroscan mode. The emission was fitted with a single exponential decay giving a lifetime of 440 ps (Figure S12).

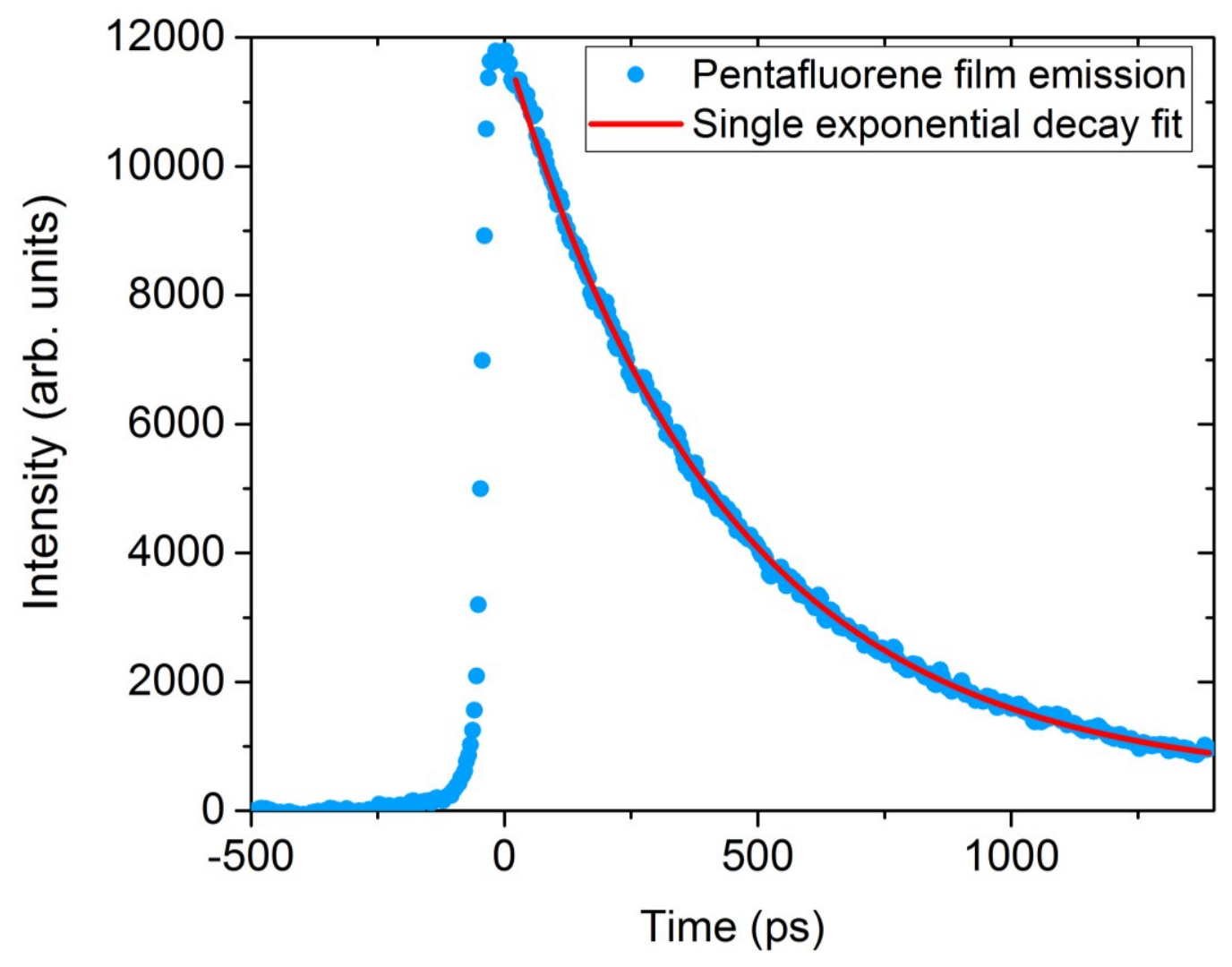

Figure S12: Experimentally recorded time resolved emission from pentafluorene film (scattered blue plot) overlapped with a single exponential decay fit (solid red line).

\section{References:}

[1] H. Suzuki, M. Hikita, Appl. Phys. Lett. 1996, 68, 2276.

[2] L. S. Hung, L. S. Liao, C. S. Lee, S. T. Lee, J. Appl. Phys. 1999, 86, 4607.

[3] H. Fujimoto, T. Miyayama, N. Sanada, C. Adachi, Org. Electron. physics, Mater. Appl. 2013, 14, 2994.

[4] A. Holländer, M. Haupt, C. Oehr, Plasma Process. Polym. 2007, 4, 773.

[5] M. Shibata, Y. Sakai, D. Yokoyama, J. Mater. Chem. C 2015, 3, 11178. 


\section{WILEY-VCH}

[6] L. Zhao, T. Komino, M. Inoue, J.-H. Kim, J. C. Ribierre, C. Adachi, Appl. Phys. Lett. 2015, 106, 063301.

[7] R. J. Holmes, S. R. Forrest, Org. Electron. 2007, 8, 77.

[8] M. S. Skolnick, T. A. Fisher, D. M. Whittaker, Semicond. Sci. Technol. 1998, 13, 645. 